\title{
Supercritical Hopf bifurcation and Turing patterns for an activator and inhibitor model with different sources
}

\author{
Jinliang Wang ${ }^{1}$, You Li $i^{*}$ (D) and Xiaojie Hou²
}

\section{"Correspondence:}

by1409118@buaa.edu.cn

'LMIB \& School of Mathematics and

System Science, Beihang University,

Beijing, P.R. China

Full list of author information is

available at the end of the article

\begin{abstract}
We study the pattern generating mechanism of a generalized Gierer-Meinhardt model with diffusions. We show the existence and stability of the Hopf bifurcation for the corresponding kinetic system under certain conditions. With spatial uneven diffusions, the obtained stable Hopf periodic solution may become unstable, which results in Turing instability. We derive conditions for the existence of Turing instability. Numerical simulations reveal that the Turing patterns are of stripe and spot shapes. In the analysis, we use bifurcation analysis, center manifold reduction for ordinary differential equations and partial differential equations. Though the Gierer-Meinhardt system is classical, our system with more general settings has yet to be analyzed in the literature.
\end{abstract}

Keywords: Supercritical; Hopf bifurcation; Turing instability; Spatial pattern

\section{Introduction}

Pattern formation can be induced by uneven diffusions. It was first discovered by Turing [1] in the 1950s. Under given conditions, chemical compounds can interact with each other and spread in space in some ways, which result in heterogeneous spatial patterns of chemical compound or morphogen concentration [2]. This means that without diffusions, the homogenous equilibrium maintains stability to small perturbations, whereas with diffusions, the homogenous equilibrium may lose its stability, and spatial inhomogeneous patterns can emerge due to the unequal spatial diffusions. Reaction-diffusion equations and systems can characterize a substantial number of pattern-related biology phenomena. In 1972, Gierer and Meinhardt [3] constructed a prototypical activator and inhibitor model of the form

$$
\left\{\begin{array}{l}
\frac{\partial a}{\partial t}=\rho_{0} \rho+c \rho \frac{a^{r}}{h^{s}}-\mu a+D_{a} \frac{\partial^{2} a}{\partial x^{2}}, \\
\frac{\partial h}{\partial t}=c^{\prime} \rho^{\prime} \frac{a^{T}}{h^{u}}-v h+D_{h} \frac{\partial^{2} h}{\partial x^{2}}
\end{array}\right.
$$

with the following assumptions: an activator $a$ and an inhibitor $h$ acting on sources of activators and inhibitors have distributions $\rho(x)$ and $\rho^{\prime}(x)$, respectively. At time $t>0$ and spatial position $x$, the concentrations of the activator and inhibitor are expressed by $a=a(x, t)$ and $h=h(x, t)$. The terms $a^{r} / h^{s}$ and $a^{T} / h^{u}$ represent the activation and inhibi-

(c) The Author(s) 2018. This article is distributed under the terms of the Creative Commons Attribution 4.0 International License (http://creativecommons.org/licenses/by/4.0/), which permits unrestricted use, distribution, and reproduction in any medium, provided you give appropriate credit to the original author(s) and the source, provide a link to the Creative Commons license, and indicate if changes were made. 
tion of sources. The terms $\mu a$ and $v h$ represent the leakage, reuptake by source, enzyme degradation, or any of their combinations. So $\mu a$ and $v h$ are removed from $a$ and $h$, and $D_{a}$ and $D_{h}$ denote the spatial diffusion coefficients. Taking into account the actual biological significance, all the parameters are positive constants. Furthermore, to form a gradient, $r, s, T$, and $u$ must satisfy $\frac{s T}{u+1}>r-1>0$, which means that $r$ must be at least 2 if it is an integer [3]. Reaction-diffusion system (1) is seen as one of the most important systems characterizing the formation of patterns $[4,5]$.

Over the years, system (1) has attracted considerable attention. In [6], the author investigated system (1), subjected to Neumann boundary conditions on the interval $(0, \pi)$, by taking $r=T=2, s=1, u=0$ and showed that if the diffusion coefficients are selected suitably, then the homogeneous steady state and the time-dependent periodic solution can undergo Turing instability. The same system was considered but subjected to Dirichlet boundary conditions on the interval $(0, l \pi), l \in \mathbb{R}^{+}$. We refer to [7] for more detail. Furthermore, many researchers studied the system by considering the saturation of activator production. In this situation, the activated area and the total structure size are proportional [8], since the activator concentration has a maximum value. In [9], the authors investigated the same system but with saturated activator production under Neumann boundary conditions in the interval $(0, \pi)$ and showed the existence of Turing instabilities of the positive spatial homogeneous equilibrium and homogenous periodic solution. They found that there are at least two limit cycles. Spectral analysis and Floquet exponent as in $[10,11]$ are significant in analyzing the dynamical behavior of reaction-diffusion systems. In [12], the authors studied the global attractivity of equilibrium and gene expression time delays in the same system with production saturation. In [13], the authors investigated system (1) with $r=s=2, T=1, u=0$ and obtained the parameter range for the system to become diffusively unstable. By taking $u=s=4$ and $r=T=2$ system (1) can be extended to the generalized or modified Gierer-Meinhardt model as in [14-17]. A meaningful result of the generalized system is the spike solutions for the related elliptic system in regions in $\mathbb{R}^{n}$. When $r=T, s=u$, and $\rho=\rho^{\prime}$, system (1) is called the general form of activator-inhibitor system with common sources [3], which was studied in [18]. They obtained a precise parametric condition for the presence of Turing instability. For more results about reaction diffusion systems of activator-inhibitor type, see [19-21]. See [22-24] and the references therein for more real-world models on Turing instability.

So far, most work on Turing instability of system (1) has been carried out by taking specific parameter values. In contrast, few work is done directly from the general system (1). The increasing number of parameters in the general system (1) make the mechanism of spatial pattern formation more difficult to understand. System (1) with $s \neq u$ is called the general form of activator-inhibitor system with different sources [3]. In comparison with the activator-inhibitor system with common sources, a system with different sources has stronger nonlinearity. Therefore, the analysis is more involved.

If $r \geq 2, r \in \mathbb{N}^{+}, r=s=T$, and $u=0$, then we obtain a class of activator-inhibitor models with different sources:

$$
\left\{\begin{array}{l}
\frac{\partial a}{\partial t}=\rho_{0} \rho+c \rho \frac{a^{r}}{h^{r}}-\mu a+D_{a} \frac{\partial^{2} a}{\partial x^{2}}, \\
\frac{\partial h}{\partial t}=c^{\prime} \rho^{\prime} a^{r}-v h+D_{h} \frac{\partial^{2} h}{\partial x^{2}} .
\end{array}\right.
$$

In this paper, we are interested in deriving the precise parametric ranges for pattern formation. We want to understand the dynamics responsible for the spot and stripe patterns. 
For simplicity, set

$$
\begin{array}{ll}
A=\left(\frac{\left(c^{\prime} \rho^{\prime}\right)^{r}}{c \rho D_{a}^{r-1}}\right)^{\frac{1}{r^{2}-r+1}} a, & H=\frac{D_{a}}{c^{\prime} \rho^{\prime}}\left(\frac{\left(c^{\prime} \rho^{\prime}\right)^{r}}{c \rho D_{a}^{r-1}}\right)^{\frac{r}{r^{2}-r+1}} h, \quad d^{*}=\frac{D_{h}}{D_{a}}, \\
\mu^{*}=\frac{\mu}{D_{a}}, \quad \nu^{*}=\frac{v}{D_{a}}, & t^{*}=D_{a} t, \quad c^{*}=\left(\frac{\left(c^{\prime} \rho^{\prime}\right)^{r}}{c \rho D_{a}^{r-1}}\right)^{\frac{1}{r^{2}-r+1}} \frac{\rho \rho_{0}}{D_{a}} .
\end{array}
$$

We drop the asterisks and use lowercase letters for algebraic convenience. Then system (2) becomes

$$
\left\{\begin{array}{l}
\frac{\partial a}{\partial t}=c+\frac{a^{r}}{h^{r}}-\mu a+\frac{\partial^{2} a}{\partial x^{2}}, \\
\frac{\partial h}{\partial t}=a^{r}-v h+d \frac{\partial^{2} h}{\partial x^{2}},
\end{array}\right.
$$

where $c, r, \mu, v, d>0, a \geq 0, h>0$, and $r \geq 2, r \in \mathbb{N}^{+}$. We also impose the Neumann boundary conditions

$$
\frac{\partial a}{\partial x}(0, t)=\frac{\partial a}{\partial x}(\pi, t)=0, \quad \frac{\partial h}{\partial x}(0, t)=\frac{\partial h}{\partial x}(\pi, t)=0
$$

to system (3).

The remaining parts of the paper are organized as follows. In Sect. 2, we proceed with a detailed study on the dynamical behavior of the corresponding kinetic system, such as the existence and stability of positive homogeneous solutions and a steady-state and timedependent periodic solution bifurcated from Hopf bifurcation. In Sect. 3, we derive sufficient analytic conditions for homogeneous solutions to undergo diffusion-driven instability. Numerical examples to illustrate the analytic results are presented in Sect. 4. From the simulations we see spot and stripe spatial patterns.

\section{Homogeneous equilibria and stability}

In this section, we investigate the existence and uniqueness of positive equilibrium for system (3) with no diffusions. System (3) with no diffusions is

$$
\left\{\begin{array}{l}
\frac{\mathrm{d} a}{\mathrm{~d} t}=c+\frac{a^{r}}{h^{r}}-\mu a, \\
\frac{\mathrm{d} h}{\mathrm{~d} t}=a^{r}-v h
\end{array}\right.
$$

For latter reference, we set

$$
f(a, h)=c+\frac{a^{r}}{h^{r}}-\mu a, \quad g(a, h)=a^{r}-v h .
$$

Then the equilibrium of system (5) satisfies

$$
f(a, h)=0, \quad g(a, h)=0 .
$$

The following lemma is needed for equilibrium analysis.

Lemma 2.1 For $c, \mu, v>0$ and $r \geq 2, r \in \mathbb{N}^{+}$, the auxiliary function $\phi(a)=c-a \mu+a^{r-r^{2}} v^{r}$, $a \in(0,+\infty)$ has the following properties: 
(1) $\phi(a)$ is a decreasing function in $(0,+\infty)$;

(2) $\lim _{a \rightarrow 0} \phi(a)=+\infty, \lim _{a \rightarrow+\infty} \phi(a)=-\infty$;

(3) $\phi(a)$ is a concave function in $(0,+\infty)$;

(4) $\phi\left(\right.$ a) has a unique zero point in $\left(\frac{c}{\mu},+\infty\right)$.

Proof Conclusions (1), (2), and (3) are obvious. The fact that $\phi\left(\frac{c}{\mu}\right)=\left(\frac{c}{\mu}\right)^{r-r^{2}} v^{r}>0$, along with (1) and (2), leads to (4).

The following proposition gives the existence and uniqueness of the positive equilibrium for system (5).

Proposition 2.1 For $a \geq 0, h>0$, system (5) has a unique positive equilibrium $\left(a_{*}, h_{*}\right)$ with $\phi\left(a_{*}\right)=0, h_{*}=a_{*}^{r} / \nu$, and $a_{*} \in\left(\frac{c}{\mu},+\infty\right)$.

Proof The equilibrium $\left(a_{*}, h_{*}\right)$ of system (5) is the solution of equation (7). Then $a_{*}>0$ and $h_{*}>0$. The remaining conclusions follow from Lemma 2.1 .

On the stability of the equilibrium $\left(a_{*}, h_{*}\right)$, we have the following:

Proposition 2.2 The unique positive equilibrium $\left(a_{*}, h_{*}\right)$ of system (5) is stable if one of the following conditions holds:

(H1) $0<(r-1) \mu \leq v, c>0$;

(H2) $(r-1) \mu>v>0, c>c_{h}$.

It is unstable if

(H3) $(r-1) \mu>v>0,0<c<c_{h}$, where $c_{h}=\frac{a_{0}}{r}[(r-1) \mu-v]$ and $a_{0}=\left(\frac{r v^{r}}{\mu+v}\right)^{\frac{1}{1-r+r^{2}}}$.

Proof Evaluated at $\left(a_{*}, h_{*}\right)$, the Jacobian matrix of (5) is

$$
\left(\begin{array}{cc}
-\mu+r v^{r} a_{*}^{-1+r-r^{2}} & -r v^{1+r} a_{*}^{-r^{2}} \\
r a_{*}^{-1+r} & -v
\end{array}\right):=J(c),
$$

so the determinant and the trace are $\operatorname{Det} J(c)=\mu \nu+(r-1) r v^{1+r} a_{*}^{-1+r-r^{2}}>0$ and $\operatorname{Tr} J(c)=$ $-\mu-v+r v^{r} a_{*}^{-1+r-r^{2}}$. The corresponding eigenvalues are

$$
\lambda_{1,2}=\frac{\operatorname{Tr} J(c) \pm \sqrt{[\operatorname{Tr} J(c)]^{2}-4 \operatorname{Det} J(c)}}{2}
$$

Denote $a_{0}=\left(\frac{r v^{r}}{\mu+v}\right)^{\frac{1}{1-r+r^{2}}}>0$. Then $\phi\left(a_{0}\right)=c-a_{0} \mu+a_{0}^{r-r^{2}} v^{r}=c+\frac{a_{0}}{r}[v-(r-1) \mu]$. If (H1) holds, then $\phi\left(a_{0}\right) \geq c>0$. From Lemma 2.1 and Proposition 2.1 we have $a_{*}>a_{0}$. Since $-1+r-r^{2}<0$, we have $a_{*}^{-1+r-r^{2}}<a_{0}^{-1+r-r^{2}}=\frac{\mu+v}{r v^{r}}$, which implies that $\operatorname{Tr} J(c)<-\mu-v+$ $r v^{r} a_{0}^{-1+r-r^{2}}=0$. Since Det $J(c)>0$, the real parts of both eigenvalues are negative. This implies that the equilibrium is stable. If (H2) holds, we have $\phi\left(a_{0}\right)>c_{h}+\frac{a_{0}}{r}[v-(r-1) \mu]=0$. From Lemma 2.1 and Proposition 2.1 we have $a_{*}>a_{0}$. Following the same analysis process as for condition (H1), we verify that the real parts of both eigenvalues of $J(c)$ are negative. Therefore, the equilibrium is stable. If (H3) holds, then from Lemma 2.1 and Proposition 2.1 we have $\phi\left(a_{0}\right)<0$ and $0<a_{*}<a_{0}$, which implies that $\operatorname{Tr} J(c)>0$. Since $\operatorname{Det} J(c)>0$, 
then one or more eigenvalues have a positive real part. This implies that the equilibrium is unstable.

The next corollary gives detailed information about the equilibrium.

Corollary 2.1 Suppose $r \geq 2$ and let

$$
\begin{array}{ll}
\mu_{01}=\frac{v\left(2 r^{2}-r+1\right)-2 v r \sqrt{r^{2}-r+1}}{(r-1)^{2}}, & \mu_{02}=\frac{v\left(2 r^{2}-r+1\right)+2 v r \sqrt{r^{2}-r+1}}{(r-1)^{2}}, \\
M_{01}=\frac{\mu-v+2 r v-2 \sqrt{r v(\mu-v+r v)}}{r v^{r}}, & \bar{M}_{01}=M_{01}^{\frac{1}{-1+r-r^{2}}}\left(\mu-M_{01} v^{r}\right), \\
M_{02}=\frac{\mu-v+2 r v+2 \sqrt{r v(\mu-v+r v)}}{r v^{r}}, & \bar{M}_{02}=M_{02}^{\frac{1}{-1+r-r^{2}}}\left(\mu-M_{02} v^{r}\right) .
\end{array}
$$

(1) For $\mu \neq v$, the equilibrium $\left(a_{*}, h_{*}\right)$ of system (5) is a stable node if one of the following conditions holds:

(SN1) $0<\mu \leq \mu_{01}, c>0$;

(SN2) $\mu>\mu_{01}, c>\bar{M}_{01}$.

It is an unstable node if

(UN) $\mu>\mu_{02}, 0<c<\bar{M}_{02}$.

(2) For $\mu \neq v$, the equilibrium $\left(a_{*}, h_{*}\right)$ of system (5) is a stable focus if one of the following conditions holds:

(SF1) $\mu_{01}<\mu \leq \frac{v}{r-1}, 0<c<\bar{M}_{01}$;

(SF2) $\mu>\frac{v}{r-1}, c_{h}<c<\bar{M}_{01}$.

It is an unstable focus if one of the following conditions holds:

(UF1) $\frac{\nu}{r-1}<\mu \leq \mu_{02}, 0<c<c_{h}$;

(UF2) $\mu>\mu_{02}, \bar{M}_{02}<c<c_{h}$.

(3) For $\mu=v$, the equilibrium $\left(a_{*}, h_{*}\right)$ of system (5) can only be a focus. It is stable if

(SF3) $c>c_{h}$

and is unstable if

(UF3) $0<c<c_{h}$.

From the analysis we can deduce from Proposition 2.2 that, for $(r-1) \mu>v>0, J\left(c_{h}\right)$ has a pair of conjugate pure imaginary eigenvalues, which implies the existence of conjugate complex eigenvalues for $c$ close to $c_{h}$. This is a necessary condition for the occurrence of the Hopf bifurcation. We further prove this and analyze the direction and stability of the Hopf bifurcation. Suppose that $(r-1) \mu>v>0$ and $c$ close to $c_{h}$, and let $x=a-a_{*}$, $y=h-h_{*}$. Then system (5) becomes

$$
\left(\begin{array}{l}
\frac{d x}{d t} \\
\frac{d y}{d t}
\end{array}\right)=J(c)\left(\begin{array}{l}
x \\
y
\end{array}\right)+\left(\begin{array}{l}
f_{2}(x, y, c) \\
g_{2}(x, y, c)
\end{array}\right),
$$

where $J(c)$ is defined as before, and

$$
f_{2}(x, y, c)=\frac{(r-1) r v^{r}}{2 a_{*}^{2-r+r^{2}}} x^{2}-\frac{r^{2} v^{1+r}}{a_{*}^{1+r^{2}}} x y+\frac{(1+r) r v^{2+r}}{2 a_{*}^{r+r^{2}}} y^{2}+\frac{(r-2)(r-1) r v^{r}}{6 a_{*}^{3-r+r^{2}}} x^{3}
$$




$$
\begin{gathered}
-\frac{(r-1) r^{2} v^{r+1}}{2 a_{*}^{2+r^{2}}} x^{2} y+\frac{(1+r) r^{2} v^{2+r}}{2 a_{*}^{1+r+r^{2}}} x y^{2}-\frac{(2+r)(1+r) r v^{3+r}}{6 a_{*}^{2 r+r^{2}}} y^{3}+O(4), \\
g_{2}(x, y, c)=\frac{1}{2}(r-1) r a_{*}^{-2+r} x^{2}+\frac{1}{6}(r-2)(r-1) r a_{*}^{-3+r} x^{3}+O(4) ;
\end{gathered}
$$

the terms with order greater than or equal to four are represented by $O(4)$. For $c=c_{h}$, we have $\lambda_{1,2}\left(c_{h}\right)= \pm i \omega_{0}$, and the eigenvector corresponding to $i \omega_{0}$ is $\xi=\left(v(\mu+v) a_{0}^{1-r}, v-i \omega_{0}\right)^{T}$, where $\omega_{0}=\sqrt{v(r \mu-v+r v)}$. Furthermore, since $r \geq 2, r \in \mathbb{N}^{+}$, we have

$$
\frac{\mathrm{d}}{\mathrm{d} c} \operatorname{Re} \lambda_{1,2}\left(c_{h}\right)=-\frac{\left(1-r+r^{2}\right)(\mu+v)}{2 a_{0}[r \mu+(r-1) \nu]}<0 .
$$

Set $x=v(\mu+v) a_{0}^{1-r} u, y=-\omega_{0} u+v v$. Then system (8) becomes

$$
\left(\begin{array}{l}
\frac{\mathrm{d} u}{\mathrm{~d} t} \\
\mathrm{~d} v \\
\mathrm{~d} t
\end{array}\right)=\left(\begin{array}{cc}
0 & -\omega_{0} \\
\omega_{0} & 0
\end{array}\right)\left(\begin{array}{l}
u \\
v
\end{array}\right)+\left(\begin{array}{l}
f_{3}\left(u, v, c_{h}\right) \\
g_{3}\left(u, v, c_{h}\right)
\end{array}\right),
$$

where

$$
\left(\begin{array}{l}
f_{3}\left(u, v, c_{h}\right) \\
g_{3}\left(u, v, c_{h}\right)
\end{array}\right)=P\left(\begin{array}{l}
f_{2}\left(r v^{r+1} a_{0}^{-r^{2}} v,-\omega_{0} u+v v, c_{h}\right) \\
g_{2}\left(r v^{r+1} a_{0}^{-r^{2}} v,-\omega_{0} u+v v, c_{h}\right)
\end{array}\right)
$$

with $P=\frac{a_{0}^{r^{2}}}{\omega_{0} v^{r+1}}\left(\begin{array}{cc}v & \frac{-r v^{r+1}}{\omega_{0}} \\ a_{0}^{r^{2}}\end{array}\right)$.

Denote

$$
\begin{aligned}
\eta= & \frac{1}{16}\left(f_{3 u u u}+g_{3 u u v}+f_{3 u v v}+g_{3 v v v}\right) \\
& +\frac{1}{16 \omega_{0}}\left[f_{3 u v}\left(f_{3 u u}+f_{3 v v}\right)-g_{3 u v}\left(g_{3 u u}+g_{3 v v}\right)-f_{3 u u} g_{3 u u}+f_{3 v v} g_{3 v v}\right]
\end{aligned}
$$

where all the partial derivatives are evaluated at the bifurcation point $(u, v, c)=\left(0,0, c_{h}\right)$. The stability of the periodic solution bifurcated from the equilibrium through Hopf bifurcation for system (5) depends on whether $\eta$ is positive or negative $[25,26]$. We calculate:

$$
\begin{aligned}
& f_{3 u и u}=(1+r)(2+r) v^{3} a_{0}^{-2 r} \omega_{0}^{2}, \quad f_{3 u v v}=v^{3} a_{0}^{-2 r}\left(-r \mu^{2}+r^{2} \mu^{2}-4 r \mu v+2 v^{2}\right), \\
& g_{3 u u}=(1+r) v a_{0}^{-r} \omega_{0}^{2}, \quad f_{3 u v}=v^{2} a_{0}^{-r}(r \mu-v), \quad g_{3 v v}=\mu v a_{0}^{-r}(r \mu-\mu-2 v), \\
& g_{3 v v v}=\mu v^{2} a_{0}^{-2 r}\left(2 \mu^{2}-3 r \mu^{2}+r^{2} \mu^{2}+6 \mu v-6 r \mu v+6 v^{2}\right), \quad f_{3 u u}=(1+r) v^{2} a_{0}^{-r} \omega_{0}, \\
& g_{3 u u v v}=(1+r)(r \mu-2 v) v^{2} a_{0}^{-2 r} \omega_{0}^{2}, g_{3 u v}=v a_{0}^{-r}(r \mu-v) \omega_{0}, \\
& f_{3 v v}=-\frac{a_{0}^{r} v^{2}}{\omega_{0}}\left[(r-1)^{2} \mu^{2}+2\left(1-r+r^{2}\right) \mu v+(r-1) r v^{2}\right],
\end{aligned}
$$

and thus

$$
\eta=-\frac{v^{3}(\mu+v)^{2}(r \mu-\mu-v)\left[\left(1+r^{2}\right) \mu+(r-1) v\right]}{16 \omega_{0}^{2} a_{0}^{2 r}}<0,
$$

since $0<v<(r-1) \mu$. A detailed information about the Hopf bifurcation is given in the following theorem. 
Theorem 2.1 For $0<v<(r-1) \mu$, system (5) undergoes a supercritical Hopf bifurcation [27] at $\left(a_{*}, h_{*}\right)$ with $c=c_{h}$ and the periodic solution bifurcated from Hopf bifurcation is stable.

\section{Analysis on the full reaction-diffusion model}

From [1] we know that the diffusion can influence the stability of the homogeneous solutions. Turing instability occurs when the homogeneous solutions, which should be stable for system (5), become unstable due to diffusions. In this section, we focus on the full reaction-diffusion model (3)-(4) to obtain the parametric ranges in which the homogeneous solutions undergo Turing instability. The analysis of Turing instabilities for the equilibrium $\left(a_{*}, h_{*}\right)$ and the periodic solution bifurcated from Hopf bifurcation is carried out respectively in Sects. 3.1 and 3.2.

\subsection{Turing instability of the equilibrium for the full reaction-diffusion model}

In this section, we follow the standard treatment for this type of problems as in [9]. First, we assume that one of conditions (H1) and (H2) holds and study system (3) with the Neumann boundary conditions (4) in the Banach space $\mathbb{H}^{2}((0, \pi)) \times \mathbb{H}^{2}((0, \pi))$, where $\mathbb{H}^{2}((0, \pi))=$ $\left\{w(\cdot) \mid \frac{\partial^{i} w}{\partial x^{i}}(\cdot) \in \mathbb{L}^{2}((0, \pi)), i=0,1,2\right\}$. Obviously, $\left(a_{*}, h_{*}\right)$ is a steady state for system (3)-(4).

Let $a=u_{1}+a_{*}, h=u_{2}+h_{*}$. The linearized system of (3) at the equilibrium $\left(a_{*}, h_{*}\right)$ is

$$
\begin{aligned}
\left(\begin{array}{c}
\frac{\partial u_{1}}{\partial t} \\
\frac{\partial u_{2}}{\partial t}
\end{array}\right) & =\left(\begin{array}{cc}
-\mu+r v^{r} a_{*}^{-1+r-r^{2}}+\partial_{x x} & -r v^{1+r} a_{*}^{-r^{2}} \\
r a_{*}^{-1+r} & -v+d \partial_{x x}
\end{array}\right)\left(\begin{array}{l}
u_{1} \\
u_{2}
\end{array}\right) \\
& :=L(c)\left(\begin{array}{l}
u_{1} \\
u_{2}
\end{array}\right) .
\end{aligned}
$$

Here the boundary conditions are:

$$
\frac{\partial u_{1}}{\partial x}(0, t)=\frac{\partial u_{1}}{\partial x}(\pi, t)=0, \quad \frac{\partial u_{2}}{\partial x}(0, t)=\frac{\partial u_{2}}{\partial x}(\pi, t)=0 .
$$

Considering the boundary conditions, the solution $\left(u_{1}, u_{2}\right) \in \mathbb{H}^{2}((0, \pi)) \times \mathbb{H}^{2}((0, \pi))$ of system (10) can formally be written as

$$
\left(\begin{array}{l}
u_{1}(x, t) \\
u_{2}(x, t)
\end{array}\right)=\sum_{k=0}^{\infty}\left(\begin{array}{l}
A_{k} \\
H_{k}
\end{array}\right) e^{\lambda_{k} t} \cos k x
$$

where $\lambda_{k} \in \mathbb{C}$ is the temporal spectrum, $k$ is the wave number, and $A_{k}, H_{k} \in \mathbb{R}$ for $k=$ $0,1,2, \ldots$ Substituting (12) into system (10), we have

$$
\sum_{k=0}^{\infty}\left(\begin{array}{l}
A_{k} \\
H_{k}
\end{array}\right) \lambda_{k} e^{\lambda_{k} t} \cos k x=\sum_{k=0}^{\infty} J_{k}(c)\left(\begin{array}{l}
A_{k} \\
H_{k}
\end{array}\right) e^{\lambda_{k} t} \cos k x,
$$

where

$$
J_{k}(c)=\left(\begin{array}{cc}
-\mu+r v^{r} a_{*}^{-1+r-r^{2}}-k^{2} & -r v^{1+r} a_{*}^{-r^{2}} \\
r a_{*}^{-1+r} & -v-d k^{2}
\end{array}\right) .
$$


Equating the like powers of $k$, we have

$$
\left(\lambda_{k} I-J_{k}(c)\right)\left(\begin{array}{l}
A_{k} \\
H_{k}
\end{array}\right)=\left(\begin{array}{l}
0 \\
0
\end{array}\right), \quad k=0,1,2, \ldots
$$

Equation (14) has a nonzero solution $\left(A_{k}, H_{k}\right)^{T}$ if and only if

$$
\operatorname{Det}\left(\lambda_{k} I-J_{k}(c)\right)=0
$$

for some $k=0,1,2, \ldots$ Namely,

$$
\lambda_{k}^{2}-\operatorname{Tr}\left(J_{k}(c)\right) \lambda_{k}+\operatorname{Det}\left(J_{k}(c)\right)=0
$$

where

$$
\operatorname{Tr}\left(J_{k}(c)\right)=-\mu-v+r v^{r} a_{*}^{-1+r-r^{2}}-(1+d) k^{2}
$$

and

$$
\operatorname{Det}\left(J_{k}(c)\right)=\left(k^{2}+\mu\right) v+(r-1) r v^{1+r} a_{*}^{-1+r-r^{2}}+d k^{2}\left[k^{2}-\left(r v^{r} a_{*}^{-1+r-r^{2}}-\mu\right)\right] .
$$

We will be concerned with the requirement $\operatorname{Re}\left(\lambda_{k}\right)>0$ on the solutions of (16).

For any wave number $k$, there exists a pair of temporal eigenvalues $\lambda_{k}^{ \pm}=$ $\frac{\operatorname{Tr}\left(J_{k}(c)\right) \pm \sqrt{\operatorname{Tr}^{2}\left(J_{k}(c)\right)-4 \operatorname{Det}\left(J_{k}(c)\right)}}{2}$. If $(\mathrm{H} 1)$ or $(\mathrm{H} 2)$ holds, then, for $k=0, \operatorname{Tr}\left(J_{k}(c)\right)=\operatorname{Tr} J(c)<0$ and $\operatorname{Det}\left(J_{k}(c)\right)=\operatorname{Det} J(c)>0$, which implies that the real parts of the related temporal spectra $\lambda_{0}^{ \pm}$are negative. Since $\operatorname{Tr}\left(J_{k}(c)\right)=\operatorname{Tr} J(c)-(1+d) k^{2}<0$ for $k=1,2, \ldots$, we need further consider the sign of $\operatorname{Det}\left(J_{k}(c)\right)$. If $r v^{r} a_{*}^{-1+r-r^{2}}-\mu \leq 1$, then we have $a_{*} \geq\left(\frac{r v^{r}}{\mu+1}\right)^{\frac{1}{1-r+r^{2}}}$ since $-1+r-r^{2}<0$. Denoting $\bar{a}=\left(\frac{r v^{r}}{\mu+1}\right)^{\frac{1}{1-r+r^{2}}}$, from Lemma 2.1 and Proposition 2.1 we have $\phi(\bar{a}) \geq 0$, that is, $c \geq \frac{\bar{a}}{r}[\mu(r-1)-1]$. Therefore $k^{2}-\left(r \nu^{r} a_{*}^{-1+r-r^{2}}-\mu\right) \geq 0$, which means that $\operatorname{Det}\left(J_{k}(c)\right)>0$. Moreover, if $m^{2}<r v^{r} a_{*}^{-1+r-r^{2}}-\mu \leq(m+1)^{2}, m \in \mathbb{N}^{+}$, then we have $\left[\frac{r v^{r}}{\mu+(m+1)^{2}}\right]^{\frac{1}{1-r+r^{2}}} \leq a_{*}<\left(\frac{r v^{r}}{\mu+m^{2}}\right)^{\frac{1}{1-r+r^{2}}}$ since $-1+r-r^{2}<0$. Denoting $a_{m+1}=\left[\frac{r v^{r}}{\mu+(m+1)^{2}}\right]^{\frac{1}{1-r+r^{2}}}$ and $a_{m}=\left(\frac{r v^{r}}{\mu+m^{2}}\right)^{\frac{1}{1-r+r^{2}}}$, from Lemma 2.1 and Proposition 2.1 we have $\phi\left(a_{m}\right)<0 \leq \phi\left(a_{m+1}\right)$, that is, $\frac{a_{m+1}}{r}\left[\mu(r-1)-(m+1)^{2}\right] \leq c<\frac{a_{m}}{r}\left[\mu(r-1)-m^{2}\right]$. In this case, for any $k=$ $m+1, m+2, \ldots$, we have $k^{2}-\left(r v^{r} a_{*}^{-1+r-r^{2}}-\mu\right) \geq 0$, which indicates that $\operatorname{Det}\left(J_{k}(c)\right)>0$, whereas for any $k=1,2, \ldots, m$, we have $k^{2}-\left(r v^{r} a_{*}^{-1+r-r^{2}}-\mu\right)<0$. Further computations reveal that if $0<d<\min _{1 \leq k \leq m} \frac{\left(k^{2}+\mu\right) v+(r-1) r v^{1+r} a_{*}^{-1+r-r^{2}}}{k^{2}\left(r v^{r} a_{*}^{-1+r-r^{2}}-\mu-k^{2}\right)}$, then $\operatorname{Det}\left(J_{k}(c)\right)>0$.

The above discussion implies that, for any spatial spectrum $k=1,2, \ldots$, the real parts of the corresponding temporal spectrum $\lambda_{k}^{ \pm}$are negative. This means the real parts of the solutions of (16) are negative for all $k=0,1,2, \ldots$, so that $\left(a_{*}, h_{*}\right)$ is asymptotically stable for (3). According to [28], because of the sectorial of $L,\left(a_{*}, h_{*}\right)$ is also locally uniformly stable, and therefore there is no Turing pattern for system (3) under the

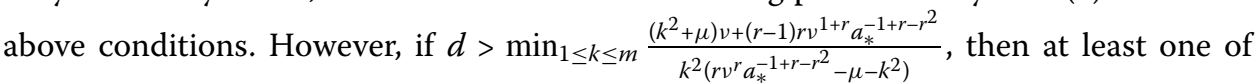
$\operatorname{Det}\left(J_{1}(c)\right), \operatorname{Det}\left(J_{2}(c)\right), \ldots, \operatorname{Det}\left(J_{m}(c)\right)$ is negative. Thus the equilibrium is unstable for (3). In this case, Turing patterns will occur. Summarizing the discussion, we have the following: 
Theorem 3.1 Let (H1) or (H2) hold. Denote

$$
\hat{d}=\min _{1 \leq k \leq m} \frac{\left(k^{2}+\mu\right) v+(r-1) r v^{1+r} a_{*}^{-1+r-r^{2}}}{k^{2}\left(r v^{r} a_{*}^{-1+r-r^{2}}-\mu-k^{2}\right)}, \quad m \in \mathbb{N}^{+} .
$$

Then the equilibrium $\left(a_{*}, h_{*}\right)$ of system (3) persists the stability if one of the following conditions holds:

(H4) $c \geq \frac{\bar{a}}{r}[\mu(r-1)-1]$

(H5) $A_{m+1} \leq c<A_{m}, 0<d<\hat{d}$.

It is unstable if

(H6) $A_{m+1} \leq c<A_{m}, d>\hat{d}$,

where $\bar{a}=\left(\frac{r v^{r}}{\mu+1}\right) \frac{1}{1-r+r^{2}}, A_{j}=\frac{a_{j}}{r}\left[\mu(r-1)-j^{2}\right], a_{j}=\left(\frac{r v^{r}}{\mu+j^{2}}\right)^{\frac{1}{1-r+r^{2}}}, j=m, m+1, m \in \mathbb{N}^{+}$.

Remark 3.1 It is worth pointing out that in Theorem 3.1 if $d=\hat{d}$, then from the discussion it follows that there exists at least one $k, k=1,2, \ldots, m, m \in \mathbb{N}^{+}$, such that the linearized system of (3) at $\left(a_{*}, h_{*}\right)$ has a zero eigenvalue. At this time, the stability of $\left(a_{*}, h_{*}\right)$ for system (3) cannot be determined by the linearized system.

\subsection{Turing instability of the limit cycle for the full reaction-diffusion model}

From Theorem 2.1 note that if $0<v<(r-1) \mu$, then there is a limit cycle bifurcated from $\left(a_{*}, h_{*}\right)$ for $c$ sufficiently close to $c_{h}$. We investigate the stability of the limit cycle obtained in Theorem 2.1. Throughout this section, we assume condition (H3), so that the limit cycle is stable to homogeneous perturbation.

Let $a=u_{1}+a_{*}, h=u_{2}+h_{*}, c=c_{h}, U=\left(u_{1}, u_{2}\right)^{T}, V=\left(v_{1}, v_{2}\right)^{T}$, and $W=\left(w_{1}, w_{2}\right)$. Then system (3)-(4) can be rewritten as $[29,30]$

$$
\left\{\begin{array}{l}
\frac{\partial U}{\partial t}=\left[J\left(c_{h}\right)+D\left(\begin{array}{cc}
\partial_{x x} & 0 \\
0 & \partial_{x x}
\end{array}\right] U+\tilde{F}\left(U, c_{h}\right),\right. \\
\frac{\partial U}{\partial x}(0, t)=\frac{\partial U}{\partial x}(\pi, t)=(0,0)^{T}
\end{array}\right.
$$

where

$$
J\left(c_{h}\right)=\left(\begin{array}{cc}
v & -r v^{r+1} a_{0}^{-r^{2}} \\
r a_{0}^{r-1} & -v
\end{array}\right), \quad D=\left(\begin{array}{ll}
1 & 0 \\
0 & d
\end{array}\right),
$$

$\tilde{F}\left(U, c_{h}\right)=\left(f_{2}\left(u_{1}, u_{2}, c_{h}\right), g_{2}\left(u_{1}, u_{2}, c_{h}\right)\right)^{T}$, and $f_{2}$ and $g_{2}$ are defined in (9). We write $\tilde{F}\left(U, c_{h}\right)=$ $\frac{1}{2} Q(U, U)+\frac{1}{6} C(U, U, U)+O\left(|U|^{4}\right)$, where $Q(U, V)=\left(Q_{1}(U, V), Q_{2}(U, V)\right)^{T}, C(U, V, W)=$ $\left(C_{1}(U, V, W), C_{2}(U, V, W)\right)^{T}$ with

$$
\begin{aligned}
& Q_{1}(U, V)=f_{2 u u} u_{1} v_{1}+f_{2 u v} u_{1} v_{2}+f_{2 v u} u_{2} v_{1}+f_{2 v v} u_{2} v_{2} \\
& =(\mu+v)\left[(r-1) a_{0}^{-1} u_{1} v_{1}-r v a_{0}^{-r}\left(u_{2} v_{1}+u_{1} v_{2}\right)\right. \\
& \left.+(r+1) v^{2} a_{0}^{1-2 r} u_{2} v_{2}\right] \\
& Q_{2}(U, V)=g_{2 u u} u_{1} v_{1}+g_{2 u v} u_{1} v_{2}+g_{2 v u} u_{2} v_{1}+g_{2 v v} u_{2} v_{2} \\
& =(r-1) r a_{0}^{r-2} u_{1} v_{1}, \\
& C_{1}(U, V, W)=f_{2 u u u} u_{1} v_{1} w_{1}+f_{2 u u v} u_{1} v_{1} w_{2}+f_{2 u v u} u_{1} v_{2} w_{1}+f_{2 v u u} u_{2} v_{1} w_{1}
\end{aligned}
$$




$$
\begin{aligned}
& +f_{2 u v v} u_{1} v_{2} w_{2}+f_{2 v u v} u_{2} v_{1} w_{2}+f_{2 v v u} u_{2} v_{2} w_{1}+f_{2 v v v} u_{2} v_{2} w_{2} \\
= & (\mu+v)\left[(r-2)(r-1) a_{0}^{-2} u_{1} v_{1} w_{1}+(1-r) r v a_{0}^{-1-r}\left(u_{1} v_{1} w_{2}\right.\right. \\
& \left.+u_{1} v_{2} w_{1}+u_{2} v_{1} w_{1}\right)+(1+r) r v^{2} a_{0}^{-2 r}\left(u_{1} v_{2} w_{2}+u_{2} v_{1} w_{2}\right. \\
& \left.\left.+u_{2} v_{2} w_{1}\right)-(r+1)(2+r) v^{3} a_{0}^{1-3 r} u_{2} v_{2} w_{2}\right], \\
C_{2}(U, V, W)= & g_{2 u u u} u_{1} v_{1} w_{1}+g_{2 u u v} u_{1} v_{1} w_{2}+g_{2 u v u} u_{1} v_{2} w_{1}+g_{2 v u u} u_{2} v_{1} w_{1} \\
& +g_{2 u v v} u_{1} v_{2} w_{2}+g_{2 v u v} u_{2} v_{1} w_{2}+g_{2 v v u} u_{2} v_{2} w_{1}+g_{2 v v v} u_{2} v_{2} w_{2} \\
= & (r-2)(r-1) r a_{0}^{r-3} u_{1} v_{1} w_{1},
\end{aligned}
$$

and $U, V, W \in \mathbb{H}^{2}((0, \pi)) \times \mathbb{H}^{2}((0, \pi))$.

For $c=c_{h}$, the linear operator $L$ defined in (10) is

$$
L U=\left[J\left(c_{h}\right)+D\left(\begin{array}{cc}
\partial_{x x} & 0 \\
0 & \partial_{x x}
\end{array}\right)\right] U
$$

and the corresponding adjoint operator, denoted by $L^{*}$, is

$$
L^{*} U=\left[J^{*}\left(c_{h}\right)+\left(\begin{array}{cc}
\partial_{x x} & 0 \\
0 & \partial_{x x}
\end{array}\right)\right] U
$$

where

$$
J\left(c_{h}\right)=\left(\begin{array}{cc}
v & -r v^{r+1} a_{0}^{-r^{2}} \\
r a_{0}^{r-1} & -v
\end{array}\right), \quad J^{*}\left(c_{h}\right)=\left(\begin{array}{cc}
v & r a_{0}^{r-1} \\
-r v^{r+1} a_{0}^{-r^{2}} & -v
\end{array}\right) .
$$

The inner product in $\mathbb{H}^{2}((0, \pi)) \times \mathbb{H}^{2}((0, \pi))$ is given by $\langle U, V\rangle=\frac{1}{\pi} \int_{0}^{\pi} \bar{U}^{T} V \mathrm{~d} x$ for $U, V \in$ $\mathbb{H}^{2}((0, \pi)) \times \mathbb{H}^{2}((0, \pi))$. Note that $\left\langle L^{*} U, V\right\rangle=\langle U, L V\rangle$. The linearized system of (17) evaluated at $(0,0)$ is

$$
\frac{\partial U}{\partial t}=L U
$$

with the Neumann boundary conditions

$$
\frac{\partial U}{\partial x}(0, t)=\frac{\partial U}{\partial x}(\pi, t)=(0,0)^{T} .
$$

System (18) with boundary conditions (19) has a solution, which can be formally written as

$$
U=\sum_{k=0}^{\infty}\left(\begin{array}{l}
a_{k} \\
h_{k}
\end{array}\right) e^{\lambda(k) t} \cos k x
$$

where $\lambda(k) \in \mathbb{C}$ are the temporal eigenvalues, $k$ is the wave number, and $a_{k}, h_{k} \in \mathbb{R}$ for $k=0,1,2, \ldots$. Substituting (20) into (18) and comparing the like terms about $k$, we have

$$
\left(\lambda(k) I-L_{k}\right)\left(\begin{array}{l}
a_{k} \\
h_{k}
\end{array}\right)=\left(\begin{array}{l}
0 \\
0
\end{array}\right), \quad k=0,1,2, \ldots,
$$


where

$$
L_{k}=\left(\begin{array}{cc}
v-k^{2} & -r v^{r+1} a_{0}^{-r^{2}} \\
r a_{0}^{r-1} & -v-d k^{2}
\end{array}\right)
$$

For some $k$, a sufficient and necessary condition for (21) to have a nonzero solution $\left(a_{k}, h_{k}\right)^{T}$ is $\operatorname{Det}\left(\lambda(k) I-L_{k}\right)=0$, that is,

$$
\lambda(k)^{2}-\operatorname{Tr}\left(L_{k}\right) \lambda(k)+\operatorname{Det}\left(L_{k}\right)=0, \quad k=0,1,2, \ldots,
$$

where $\operatorname{Tr}\left(L_{k}\right)=-(1+d) k^{2}$ and $\operatorname{Det}\left(L_{k}\right)=k^{2} v+v(r \mu-v+r v)+k^{2}\left(k^{2}-v\right) d$. We are interested in the solutions $\lambda(k)$ such that $\operatorname{Re}(\lambda(k))>0$.

With $0<v<(r-1) \mu$ and $c=c_{h}$, we have $\operatorname{Tr}\left(L_{0}\right)=0$, $\operatorname{Det}\left(L_{0}\right)=v(r \mu-v+r v)>0$, and $\operatorname{Tr}\left(L_{k}\right)<0$ for $k=1,2, \ldots$. This means that, for $k=0$, the real parts of the eigenvalues of $L$ are zero. We then have to do the center manifold reduction.

First of all, for $k=1,2, \ldots$, if $0<v \leq 1$, then $\operatorname{Det}\left(L_{k}\right)>0$. Furthermore, if $m^{2}<v \leq(m+1)^{2}$ and $0<d<\bar{d}$, where $\bar{d}=\min _{1 \leq k \leq m} \frac{k^{2} v+v(r \mu-v+r v)}{-k^{2}\left(k^{2}-v\right)}, m \in \mathbb{N}^{+}$, then we have $\operatorname{Det}\left(L_{k}\right)>0$ for $k=$ $1,2, \ldots$, and if $m^{2}<v \leq(m+1)^{2}$ and $d>d$, then at least one of $\operatorname{Det}\left(L_{1}\right), \operatorname{Det}\left(L_{2}\right), \ldots, \operatorname{Det}\left(L_{m}\right)$ is negative.

Letting $\xi=\left(v(\mu+v) a_{0}^{1-r}, v-i \omega_{0}\right)^{T}$ and $\xi^{*}=\frac{1}{2 \omega_{0}}\left(\frac{\omega_{0}+i v}{r v^{r+1}} a_{0}^{r^{2}},-i\right)^{T}$, we have $L \xi=i \omega_{0} \xi, L^{*} \xi^{*}=$ $-i \omega_{0} \xi^{*},\left\langle\xi^{*}, \bar{\xi}\right\rangle=0$, and $\left\langle\xi^{*}, \xi\right\rangle=1$. Let $U=z \xi+\bar{z} \bar{\xi}+w, z=\left\langle\xi^{*}, U\right\rangle, w=\left(w_{1}, w_{2}\right)^{T}$. Then

$$
\left\{\begin{array}{l}
u=v(\mu+v) a_{0}^{1-r}(z+\bar{z})+w_{1}, \\
v=\left(v-i \omega_{0}\right) z+\left(v+i \omega_{0}\right) \bar{z}+w_{2} .
\end{array}\right.
$$

In $(z, w)$ coordinates, system (17) is

$$
\left\{\begin{array}{l}
\frac{d z}{d t}=i \omega_{0} z+\left\langle\xi^{*}, \tilde{f}\right\rangle \\
\frac{d w}{d t}=L w+\tilde{H}(z, \bar{z}, w)
\end{array}\right.
$$

where $\tilde{f}=\tilde{F}\left(z \xi+\bar{z} \bar{\xi}+w, c_{h}\right)$ and $\tilde{H}(z, \bar{z}, w)=\tilde{f}-\left\langle\xi^{*}, \tilde{f}\right\rangle \xi-\left\langle\bar{\xi}^{*}, \tilde{f}\right\rangle \bar{\xi}$. From further calculation we have

$$
\begin{aligned}
\tilde{F}(z \xi+ & \left.\bar{z} \bar{\xi}+w, c_{h}\right)=\frac{1}{2} Q(\xi, \xi) z^{2}+Q(\xi, \bar{\xi}) z \bar{z}+\frac{1}{2} Q(\bar{\xi}, \bar{\xi}) \bar{z}^{2}+O\left(|z|^{3},|z| \cdot|w|,|w|^{2}\right) \\
\left\langle\xi^{*}, \tilde{f}\right\rangle= & \frac{1}{2}\left\langle\xi^{*}, Q(\xi, \xi)\right| z^{2}+\left\langle\xi^{*}, Q(\xi, \bar{\xi})\right| z \bar{z}+\frac{1}{2}\left\langle\xi^{*}, Q(\bar{\xi}, \bar{\xi})\right| \bar{z}^{2} \\
& +O\left(|z|^{3},|z| \cdot|w|,|w|^{2}\right) \\
\left\langle\bar{\xi}^{*}, \tilde{f}\right\rangle= & \frac{1}{2}\left\langle\bar{\xi}^{*}, Q(\xi, \xi)\right| z^{2}+\left\langle\bar{\xi}^{*}, Q(\xi, \bar{\xi})\right| z \bar{z}+\frac{1}{2}\left\langle\bar{\xi}^{*}, Q(\bar{\xi}, \bar{\xi})\right| \bar{z}^{2} \\
& +O\left(|z|^{3},|z| \cdot|w|,|w|^{2}\right) .
\end{aligned}
$$

So $\tilde{H}(z, \bar{z}, w)=\frac{1}{2} z^{2} \tilde{H}_{20}+z \bar{z} \tilde{H}_{11}+\frac{1}{2} \bar{z}^{2} \tilde{H}_{02}+O\left(|z|^{3},|z| \cdot|w|,|w|^{2}\right)$, where

$$
\tilde{H}_{20}=Q(\xi, \xi)-\left\langle\xi^{*}, Q(\xi, \xi)\right| \xi-\left\langle\bar{\xi}^{*}, Q(\xi, \xi)\right| \bar{\xi}
$$




$$
\begin{aligned}
= & \left(Q_{1}(\xi, \xi), Q_{2}(\xi, \xi)\right)^{T}-\frac{1}{2 \omega_{0}}\left[\frac{\omega_{0}-i v}{v(\mu+v)} a_{0}^{r-1} Q_{1}(\xi, \xi)+i Q_{2}(\xi, \xi)\right] \xi \\
& -\frac{1}{2 \omega_{0}}\left[\frac{\omega_{0}+i v}{v(\mu+v)} a_{0}^{r-1} Q_{1}(\xi, \xi)-i Q_{2}(\xi, \xi)\right] \bar{\xi} \\
= & (0,0)^{T} \\
\tilde{H}_{11}= & Q(\xi, \bar{\xi})-\left\langle\xi^{*}, Q(\xi, \bar{\xi})\right| \xi-\left\langle\bar{\xi}^{*}, Q(\xi, \bar{\xi})\right| \bar{\xi} \\
= & \left(Q_{1}(\xi, \bar{\xi}), Q_{2}(\xi, \bar{\xi})\right)^{T}-\frac{1}{2 \omega_{0}}\left[\frac{\omega_{0}-i v}{v(\mu+v)} a_{0}^{r-1} Q_{1}(\xi, \bar{\xi})+i Q_{2}(\xi, \bar{\xi})\right] \xi \\
& -\frac{1}{2 \omega_{0}}\left[\frac{\omega_{0}+i v}{v(\mu+v)} a_{0}^{r-1} Q_{1}(\xi, \bar{\xi})-i Q_{2}(\xi, \bar{\xi})\right] \bar{\xi} \\
= & (0,0)^{T}, \\
\tilde{H}_{02}= & Q(\bar{\xi}, \bar{\xi})-\left\langle\xi^{*}, Q(\bar{\xi}, \bar{\xi})\right) \xi-\left\langle\bar{\xi}^{*}, Q(\bar{\xi}, \bar{\xi})\right| \bar{\xi} \\
= & \left(Q_{1}(\bar{\xi}, \bar{\xi}), Q_{2}(\bar{\xi}, \bar{\xi})\right)^{T}-\frac{1}{2 \omega_{0}}\left[\frac{\omega_{0}-i v}{v(\mu+v)} a_{0}^{r-1} Q_{1}(\bar{\xi}, \bar{\xi})+i Q_{2}(\bar{\xi}, \bar{\xi})\right] \xi \\
& -\frac{1}{2 \omega_{0}}\left[\frac{\omega_{0}+i v}{v(\mu+v)} a_{0}^{r-1} Q_{1}(\bar{\xi}, \bar{\xi})-i Q_{2}(\bar{\xi}, \bar{\xi})\right] \bar{\xi} \\
= & (0,0)^{T} .
\end{aligned}
$$

Then we have $\tilde{H}(z, \bar{z}, w)=O\left(|z|^{3},|z| \cdot|w|,|w|^{2}\right)$. System (23) has a center manifold of the form $w=\frac{w_{20}}{2} z^{2}+w_{11} z \bar{z}+\frac{w_{02}}{2} \bar{z}^{2}+O\left(|z|^{3}\right)$. With $L w+\tilde{H}(z, \bar{z}, w)=\frac{d w}{d t}=\frac{\partial w}{\partial z} \frac{d z}{d t}+\frac{\partial w}{\partial \bar{z}} \frac{d \bar{z}}{d t}$, we obtain $w_{20}=\left[2 i \omega_{0}-L\right]^{-1} \tilde{H}_{20}=(0,0)^{T}, w_{11}=-L^{-1} \tilde{H}_{11}=(0,0)^{T}, w_{02}=\left[-2 i \omega_{0}-L\right]^{-1} \tilde{H}_{02}=(0,0)^{T}$. Therefore, on the center manifold, the reaction-diffusion system is

$$
\dot{z}=i \omega_{0} z+\left\langle\xi^{*}, \tilde{f}\right\rangle=i \omega_{0} z+\sum_{2 \leq i+j \leq 3} \frac{g_{i j}}{i ! j !} z^{i} \bar{z}^{j}+O\left(\left|z^{4}\right|\right)
$$

where

$$
\begin{array}{ll}
g_{20}=\left\langle\xi^{*}, Q(\xi, \xi)\right\rangle, & g_{11}=\left\langle\xi^{*}, Q(\xi, \bar{\xi})\right\rangle, \\
g_{02}=\left\langle\xi^{*}, Q(\bar{\xi}, \bar{\xi})\right\rangle, & g_{21}=\left\langle\xi^{*}, C(\xi, \xi, \bar{\xi})\right\rangle .
\end{array}
$$

We rewrite (17) into the Poincaré normal form in a neighborhood of $c_{h}$ :

$$
\frac{\mathrm{d} z}{\mathrm{~d} t}=(\alpha(c)+i \omega(c)) z+z \sum_{j=1}^{M} \delta_{j}(c)(z \bar{z})^{j}
$$

where the variable $z \in \mathbb{C}, M \geq 1$, and the coefficients $\delta_{j}(c)$ are complex. Direct computation shows that

$$
\delta_{1}(c)=\frac{g_{20} g_{11}[3 \alpha(c)+i \omega(c)]}{2\left[\alpha^{2}(c)+\omega^{2}(c)\right]}+\frac{\left|g_{11}\right|^{2}}{\alpha(c)+i \omega(c)}+\frac{\left|g_{02}\right|^{2}}{2[\alpha(c)+3 i \omega(c)]}+\frac{g_{21}}{2}
$$


and thus $\operatorname{Re}\left(\delta_{1}\left(c_{h}\right)\right)=\operatorname{Re}\left[\frac{g_{20} g_{11}}{2 \omega_{0}} i+\frac{g_{21}}{2}\right]$. Since $\alpha\left(c_{h}\right)=0, \omega\left(c_{h}\right)=\omega_{0}>0$, and $0<\nu<(r-1) \mu$, we then have

$$
\operatorname{Re}\left[\delta_{1}\left(c_{h}\right)\right]=-\frac{[(r-1) \mu-v] v^{3}(\mu+v)^{2}\left[\left(1+r^{2}\right) \mu+(r-1) v\right]}{4 \omega_{0}^{2} a_{0}^{2 r}}<0 .
$$

Therefore, the supercritical Hopf bifurcation occurs at $c=c_{h}$. The following theorem is a summary of the preceding analysis.

Theorem 3.2 Let (H3) hold, and let

$$
\bar{d}=\min _{1 \leq k \leq m} \frac{k^{2} v+v(r \mu-v+r v)}{-k^{2}\left(k^{2}-v\right)}, \quad m \in \mathbb{N}^{+} .
$$

Then the spatially homogenous periodic solution for (3) is stable if either (H7) or (H8) holds and is unstable if (H9) holds, where

(H7) $0<v \leq 1$,

(H8) $m^{2}<v \leq(m+1)^{2}, 0<d<\bar{d}$, and

(H9) $m^{2}<v \leq(m+1)^{2}, d>\bar{d}$.

Remark 3.2 Theorem 3.1 and 3.2 show that system (3) subjected to (4) undergoes Turing instability under either of assumptions (H1) and (H6), or (H2) and (H6), or (H3) and (H9), which is responsible for the patterns of stripe and spot types. We will see these patterns numerically in the next section.

\section{Numerical simulation}

In this section, numerical examples are used to illustrate the main conclusions in Sects. 2 and 3. In this section, we assume that $r=2, c>0, v>0$, and $\mu>0$.

First of all, we illustrate the results in Proposition 2.2 and Theorem 2.1. For the dynamics of the equilibrium of the temporal model (5), we just show the simulations when (H2) and (H3) are satisfied. The simulations when condition (H1) is satisfied are omitted since the dynamic behavior is similar with the case where condition (H2) holds.

Set $v=0.5$ and $\mu=3$. Then $c_{h}=0.6534$. Let $c=0.75$, so that condition (H2) holds. The equilibrium $\left(a_{*}, h_{*}\right)$ of system (5) is $(0.538,0.5788)$. We choose the initial state $\left(a_{0}, h_{0}\right)=(0.588,0.6288)$. From the simulation we find that the orbit starting from $\left(a_{0}, h_{0}\right)=$ $(0.588,0.6288)$ converges to the equilibrium $\left(a_{*}, h_{*}\right)=(0.538,0.5788)$. The equilibrium is asymptotically stable. See Fig. 1.

Set $v=1.5$ and $\mu=3$. Then $c_{h}=0.75$. We take $c=0.74$, so that condition (H3) holds. The equilibrium $\left(a_{*}, h_{*}\right)$ of system (5) is $(0.9987,0.6649)$. The initial state $\left(a_{0}, h_{0}\right)$ we choose is $(1.0487,0.7149)$. From the simulation we find that the phase orbit represented by the blue solid line in Figs. 2 and 3, starting from $\left(a_{0}, h_{0}\right)=(1.0487,0.7149)$ goes counterclockwise away from the equilibrium $\left(a_{*}, h_{*}\right)=(0.9987,0.6649)$, so the equilibrium $\left(a_{*}, h_{*}\right)=(0.9987,0.6649)$ is unstable. Furthermore, we choose another initial state $\left(a_{0}, h_{0}\right)=(1.25,0.75)$. From the simulation we find that the phase orbit represented by the red solid line in Fig. 3, starting from $\left(a_{0}, h_{0}\right)=(1.25,0.75)$ goes counterclockwise inward. Since the orbits cannot intersect each other, there must be a limit cycle, and it is stable. 


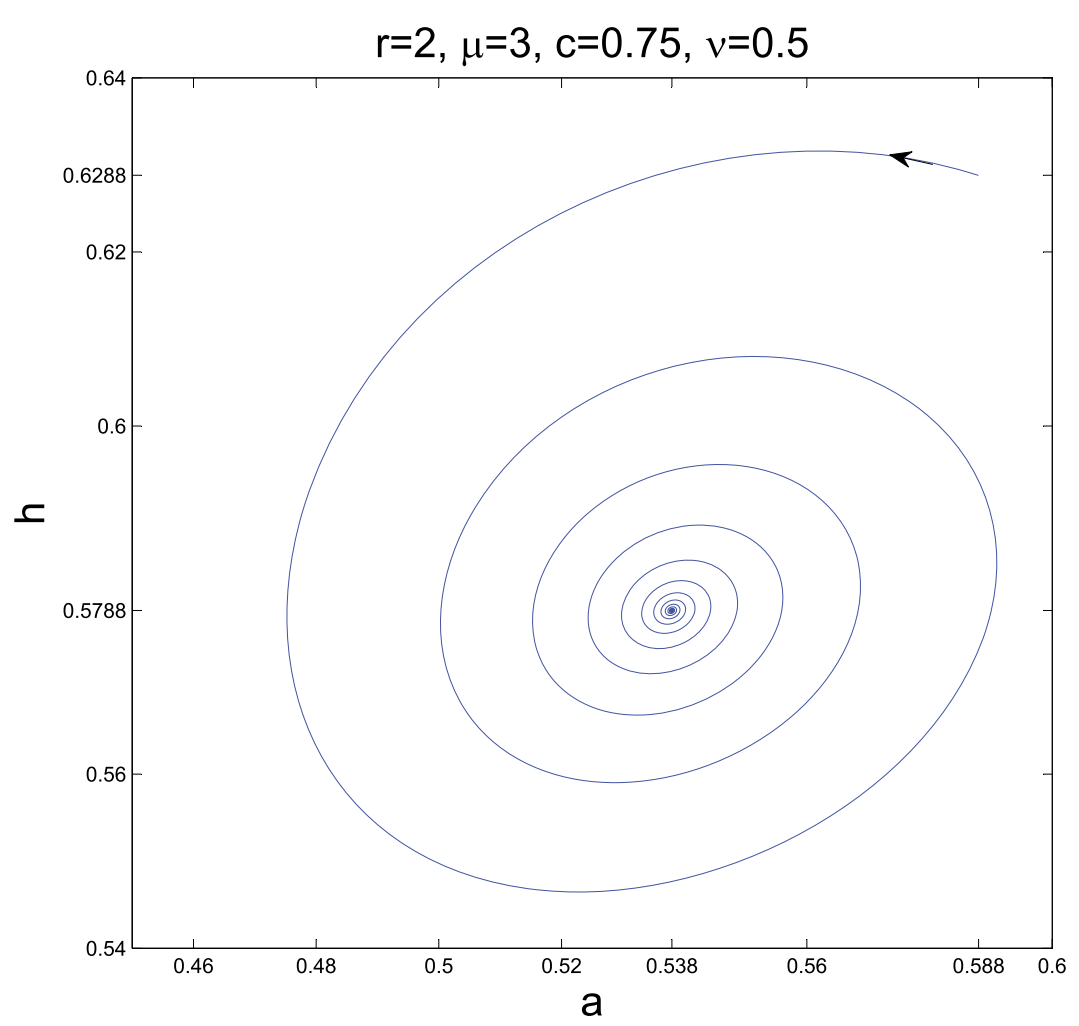

Figure 1 The stable equilibrium of (5) under condition ( $\mathrm{H} 2)$

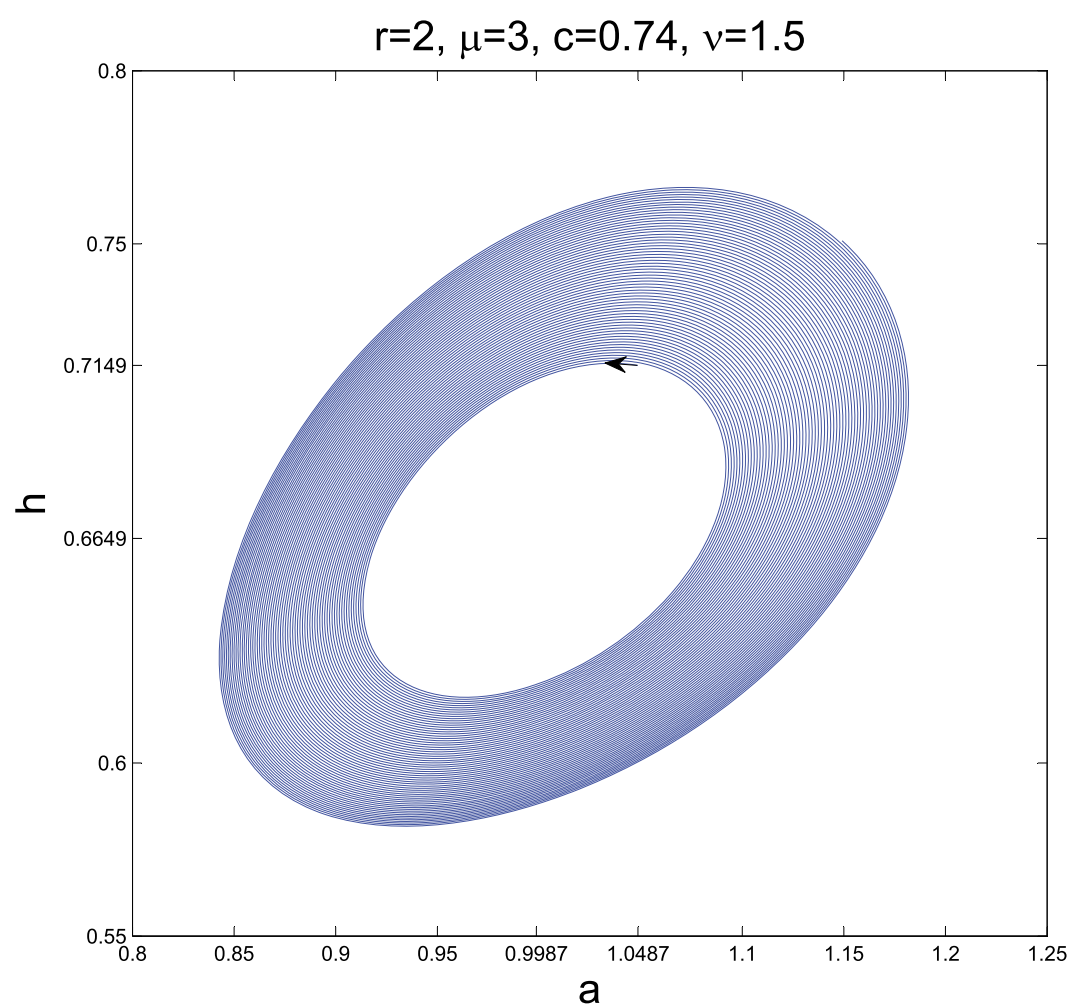

Figure 2 The unstable equilibrium of (5) under condition ( $\mathrm{H} 3$ ) 


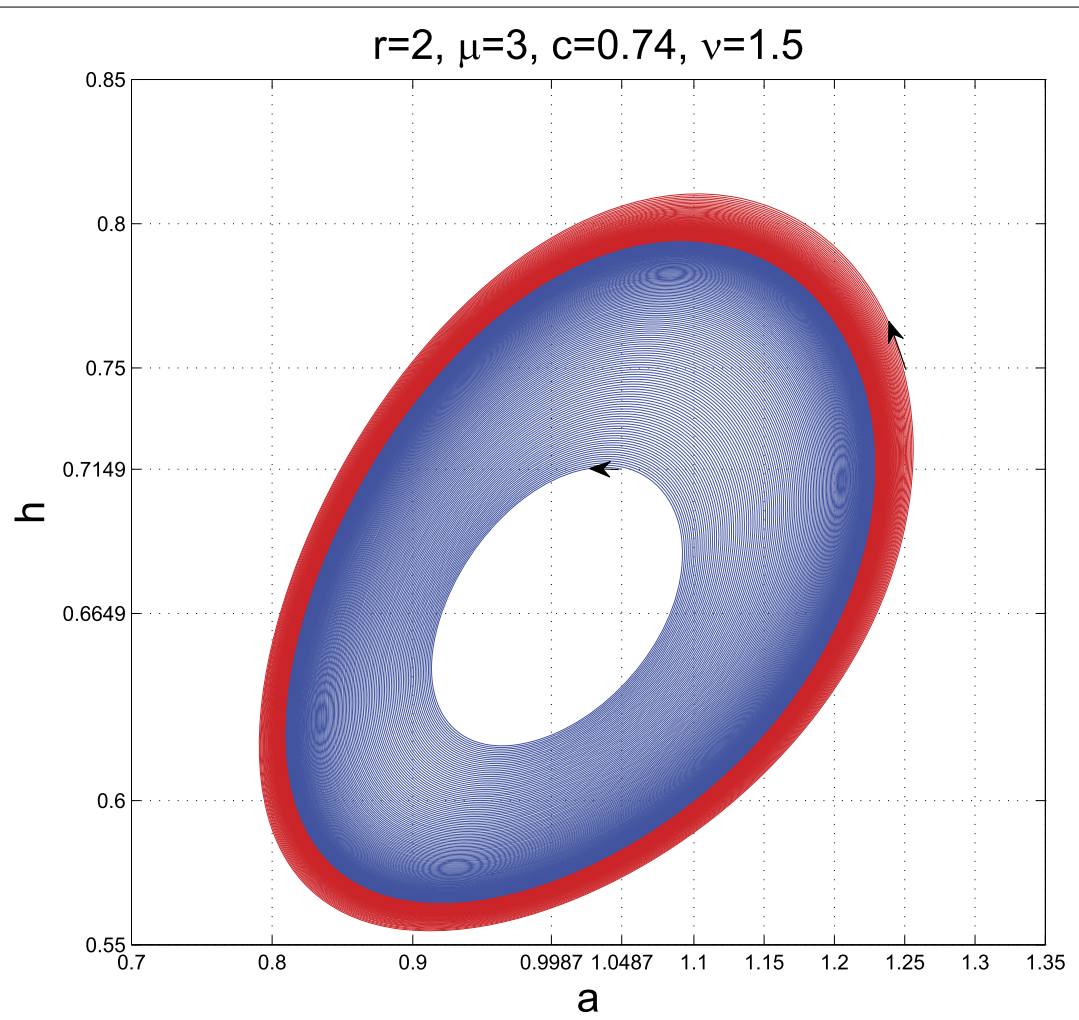

Figure 3 The orbits converse to the limit cycle. The phase orbits starting from $\left(a_{0}, h_{0}\right)=(1.0487,0.7149)$ and $\left(a_{0}, h_{0}\right)=(1.25,0.75)$ both converge to the stable limit cycle

The stable limit cycle is shown in Fig. 4. Our simulations support Proposition 2.2 and Theorem 2.1.

In addition, the supercritical Hopf bifurcation surface $c=c_{h}$ of system (5) in the parameter space $(v, \mu, c)$ and the supercritical Hopf bifurcation diagram of (5) for $\mu=3$ in parameter coordinates $(v, c)$ are shown respectively in Figs. 5 and 6.

In the following, we illustrate the results obtained in Theorem 3.1.

Let $c=0.75, v=0.5, \mu=3$, and $d=2$. Then $\bar{a}=0.5$ and $\frac{\bar{a}}{r}[\mu(r-1)-1]=0.5$, which supports conditions (H2) and (H4). The initial state we choose is $(0.53801,0.57879)$, which is a small perturbation of the equilibrium $\left(a_{*}, h_{*}\right)=(0.538,0.5788)$. The dynamics are shown in Fig. 7 . The simulation shows that the stable equilibrium $\left(a_{*}, h_{*}\right)$ does not change its stability under diffusions.

Let $c=0.76, v=1.5, \mu=3$, and $m=1$. Then $a_{1}=1.04, a_{2}=0.863, A_{1}=1.04, A_{2}=$ -0.4315 , and $\hat{d}=26.3949$. Set $d=\hat{d}-1=25.3949$. Then (H2) and (H5) hold. The equilibrium is $\left(a_{*}, h_{*}\right)=(1.0013,0.6684)$, and the initial condition is $(1.00129,0.66839)$. The corresponding dynamics are shown in Fig. 8. The equilibrium $\left(a_{*}, h_{*}\right)$ is stable for system (3).

In both cases the equilibrium is locally uniformly stable, and hence Turing patterns do not occur.

Subsequently, let $c=0.76, v=1.5, \mu=3$, and $m=1$ and set $d=\hat{d}+1=27.3949$. Then $(\mathrm{H} 2)$ and (H6) hold. The stable equilibrium $\left(a_{*}, h_{*}\right)=(1.0013,0.6684)$ for system (5) becomes unstable for system (3) due to the influence of the diffusions. The initial condition is $\left(a_{*}-0.00001 \cos (x), h_{*}-0.00001 \sin (x)\right), x \in(0,300)$. The dynamics are shown in Fig. 9. In this case, Turing patterns occur. From the projections of the dynamics for the activator 


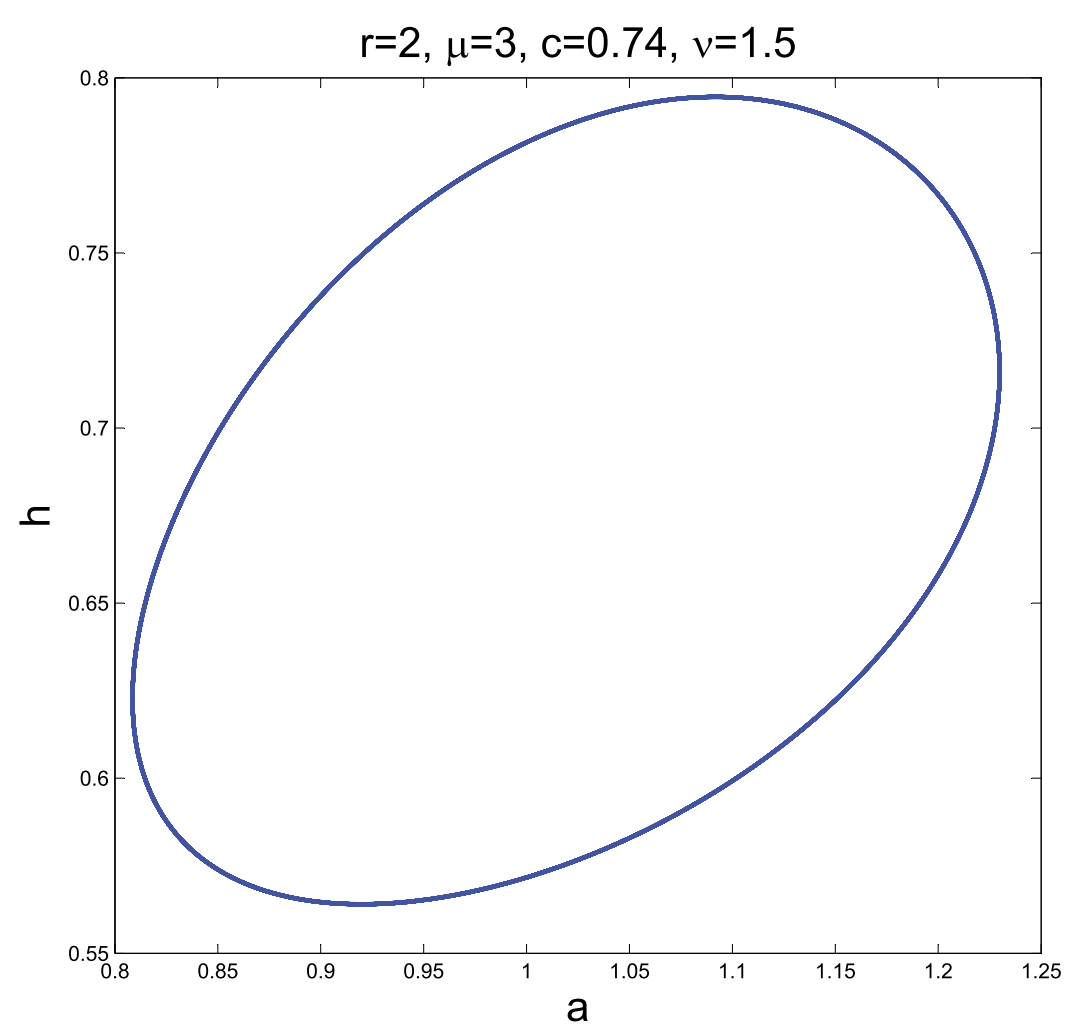

Figure 4 The stable limit cycle of (5)

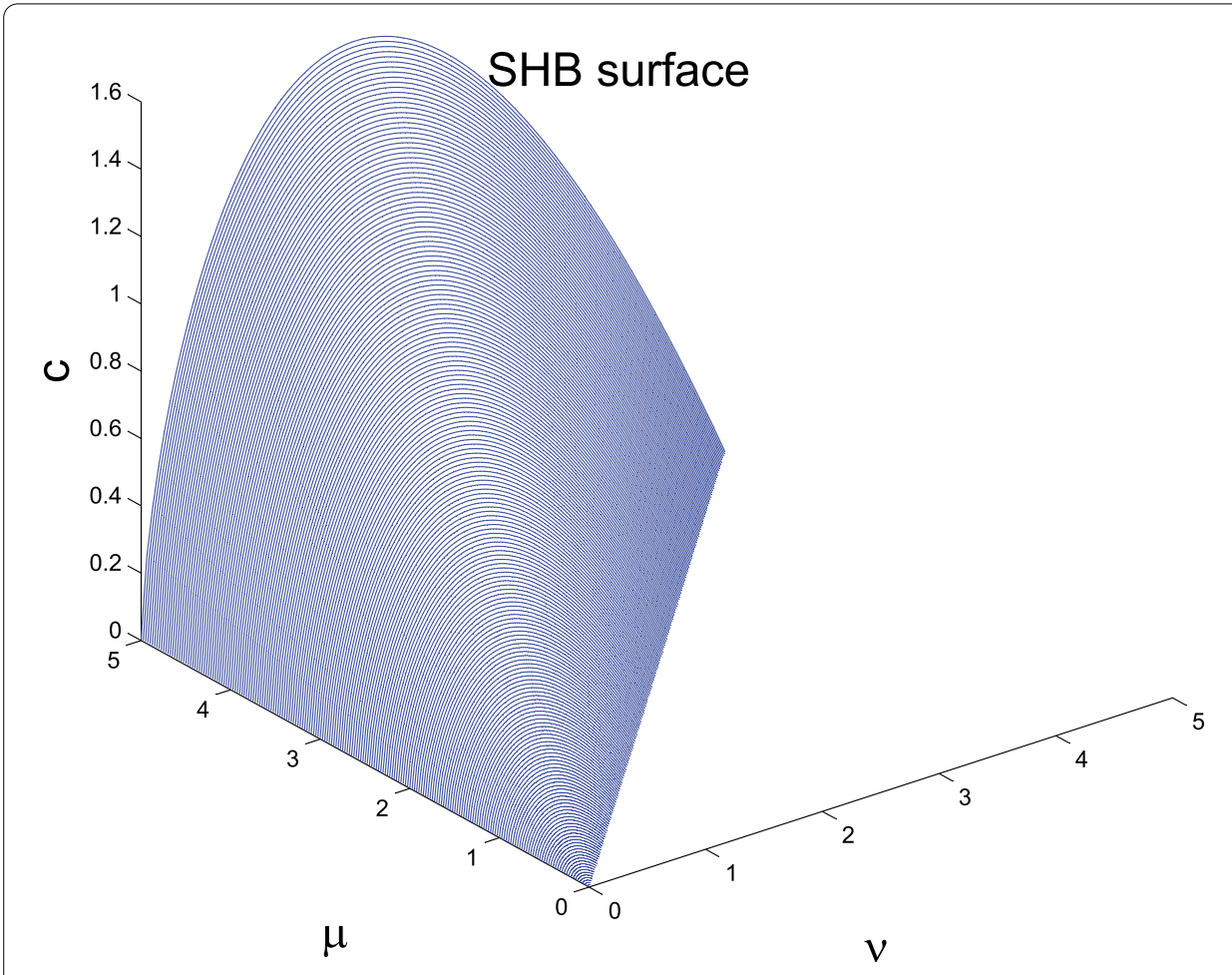

Figure 5 The supercritical Hopf bifurcation (SHB) surface of (5) in parameter space $(v, \mu, c)$ 


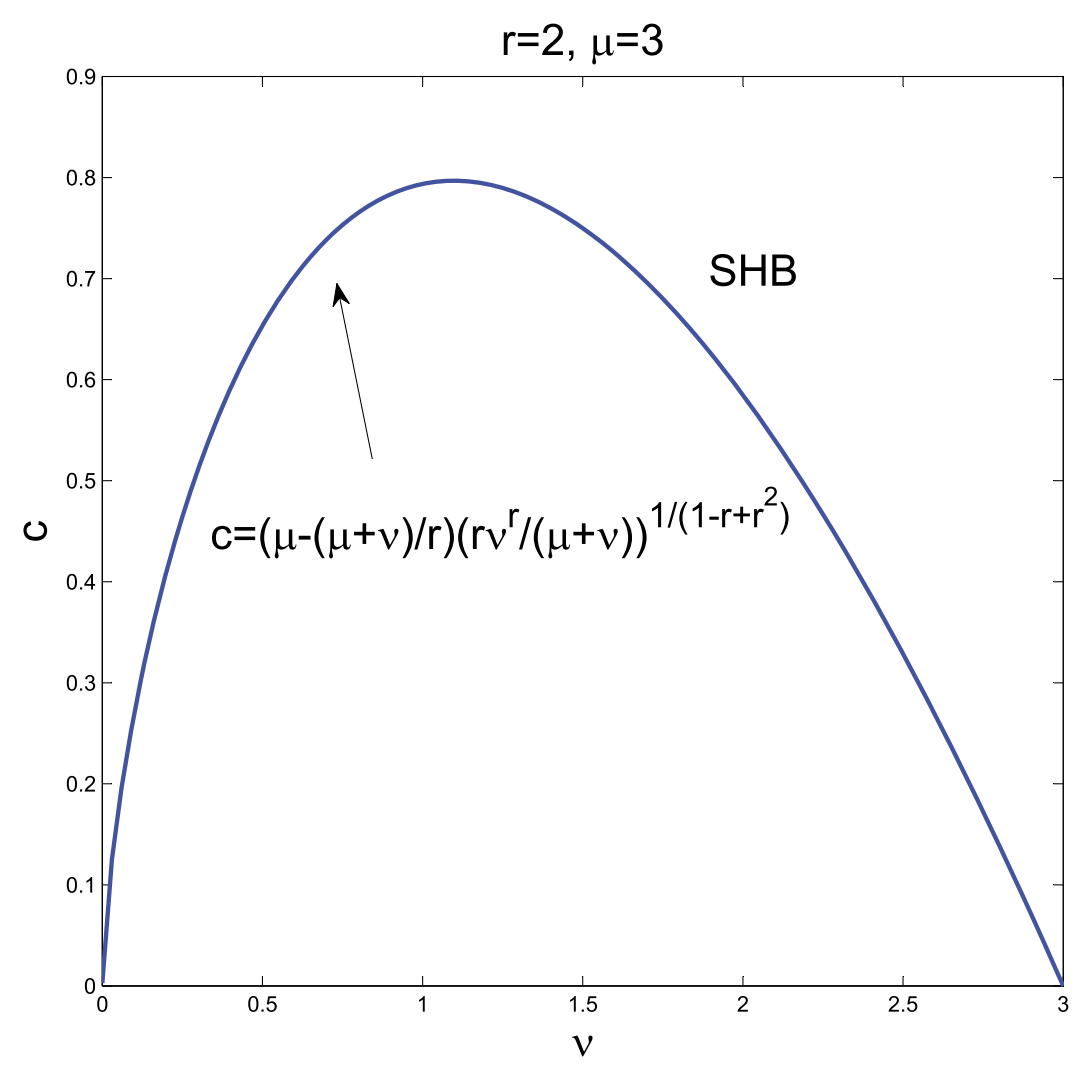

Figure 6 The Hopf bifurcation diagram of (5) for $\mu=3$ in parameter coordinates $(v, c)$

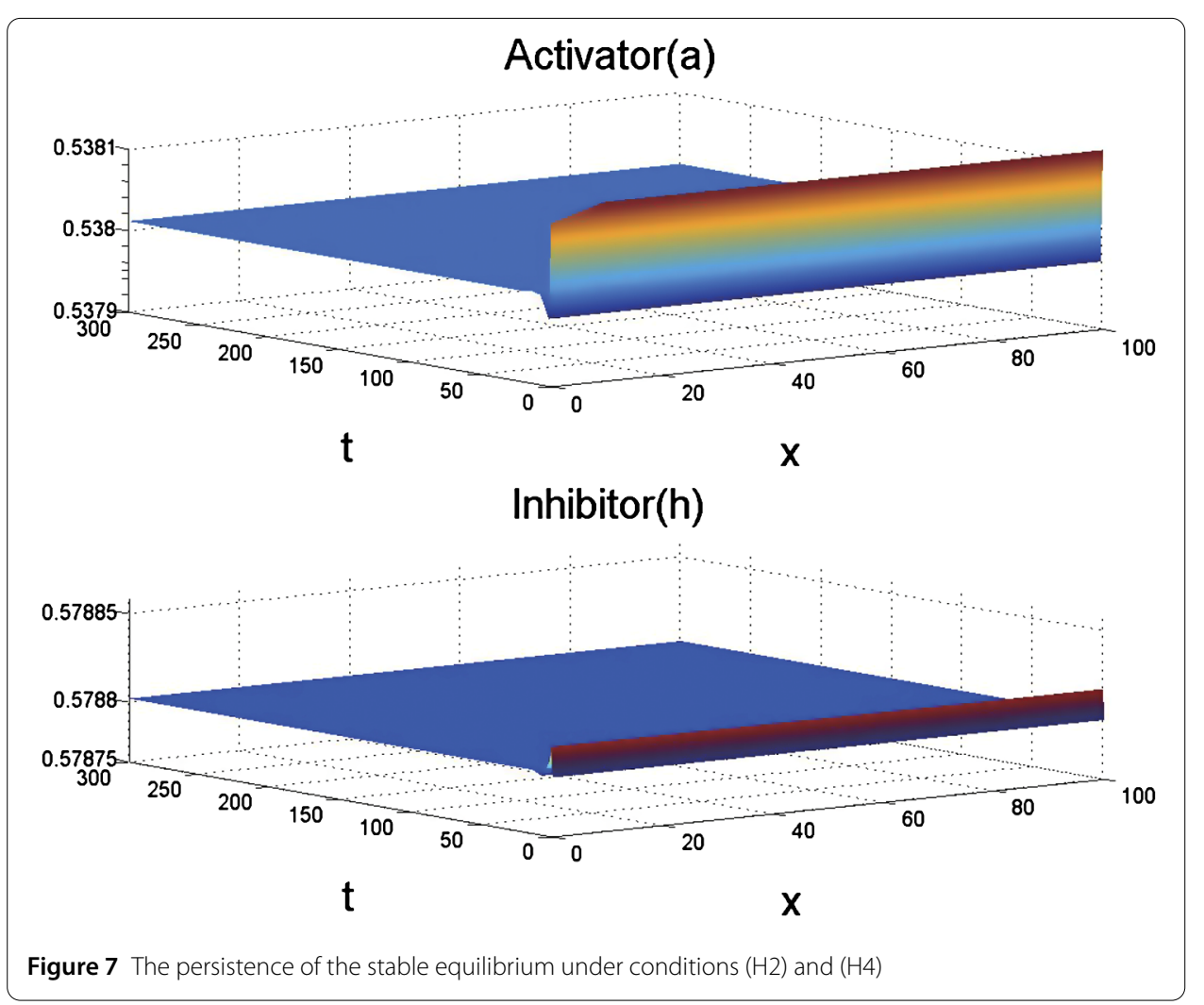



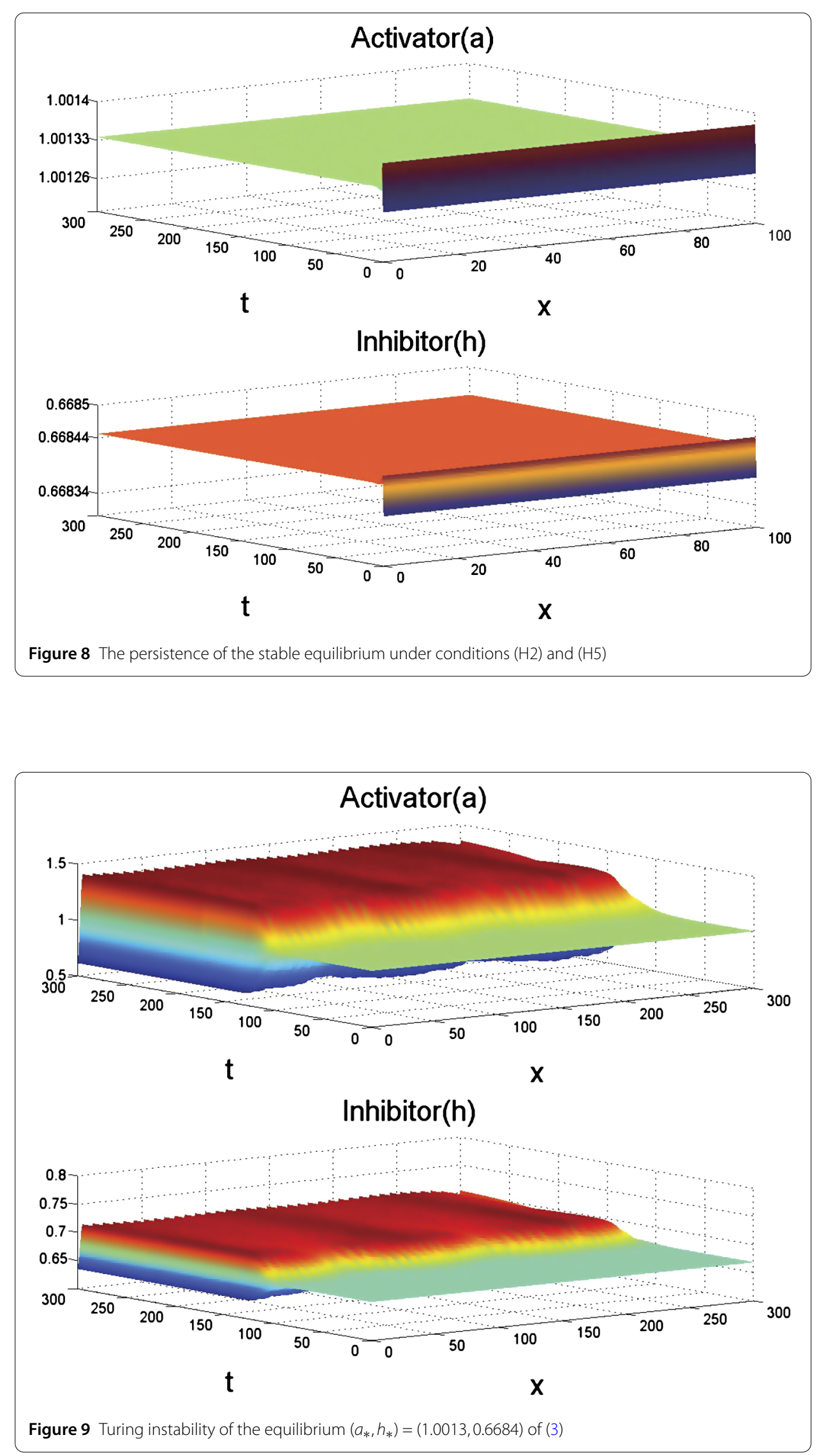

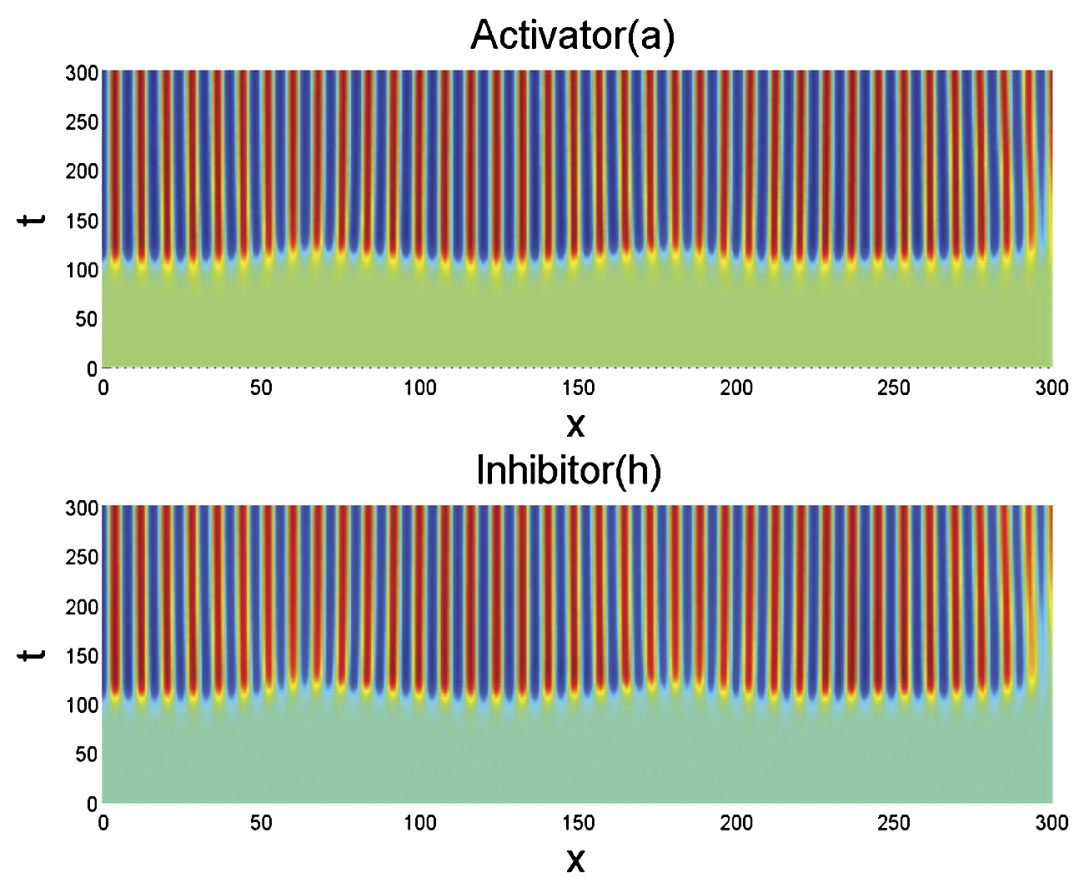

Figure 10 The projection of Fig. 9 in $(x, t)$ coordinates

$a$ and inhibitor $h$, respectively, shown in Fig. 10, we see the stripe patterns. These stripe patterns are formed because the equilibrium $\left(a_{*}, h_{*}\right)$ undergoes Turing instability.

We next illustrate the results in Theorem 3.2. In the simulation, we use the criterion in [31] for the choice of the space-time scaling parameter. From Theorem 3.2 we find that the stable limit cycle does not undergo Turing instability under (H3) and (H7) or under (H3) and (H8). Here we just illustrate the case where (H3) and (H8) hold.

Let $c=0.74, v=1.5$, and $\mu=3$, which implies that (H3) holds. Then $m=1$ and $\bar{d}=25.5$. Set $d=\bar{d}-0.5$. Then (H8) holds. According to the simulation about the limit cycle, we find a point $(0.8614,0.7902)$ on it. The initial condition is a small spatial heterogeneous perturbation of this point, namely $(0.8614+0.000001 \sin (x), 0.7092+0.00001 \cos (x)), x \in$ $(0,150)$. The dynamics are shown in Fig. 11 which implies that the stable limit cycle of kinetic system (5) is still stable for the spatial temporal system (3). Figure 12 is the projection of Fig. 11 in $(x, t)$ coordinates. In this case the limit cycle does not undergo Turing instability.

Let $c=0.74, v=1.5$, and $\mu=3$, which implies that (H3) holds. Then $m=1$ and $\bar{d}=25.5$. Set $d=\bar{d}+1.5$. Then (H9) holds. The initial condition is also $(0.8614+$ $0.000001 \sin (x), 0.7092+0.00001 \cos (x)), x \in(0,150)$. We can see that the stable limit cycle of system (5) becomes unstable for the spatial temporal system (3) because of the diffusions. In this case, the Turing instability of the limit cycle takes place; see Fig. 13. Figure 14 is a local enlargement of the projection of Fig. 13. Moreover, from the simulation we can easily see the spot patterns. These spot patterns are formed because the limit cycle undergoes Turing instability.

Furthermore, we obtain the regions where Turing patterns may occur in the parameter space $\left(D_{h}, c\right)$ by setting $r=2, \mu=3, v=1.5$; see Fig. 15 . From the above simulations we know that $c_{h}=0.75$. The regions above the line $c=c_{h}$ are divided into two parts. The pink region in which no Turing patterns are formed is for the stable equilibrium. The blue one 

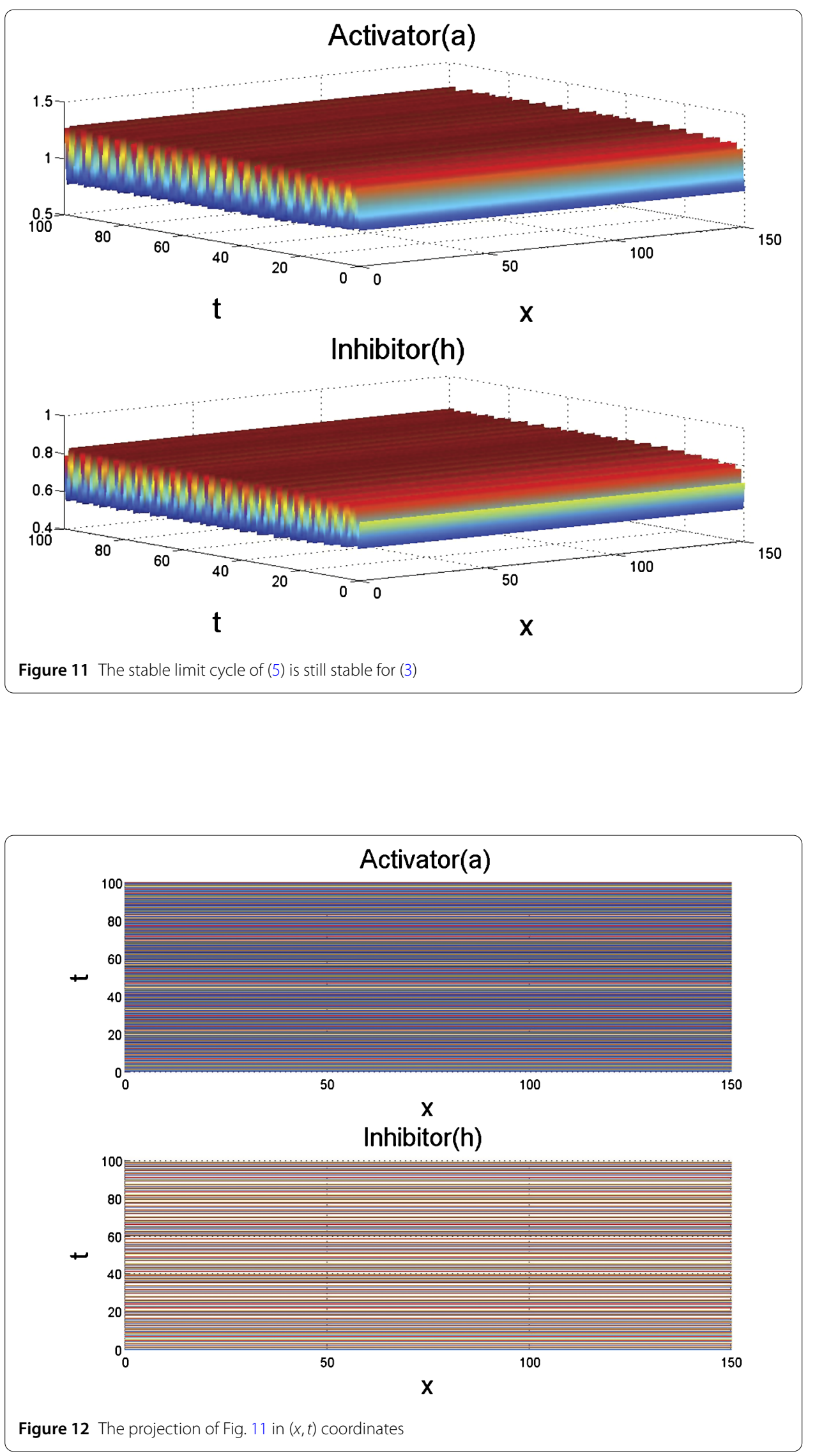

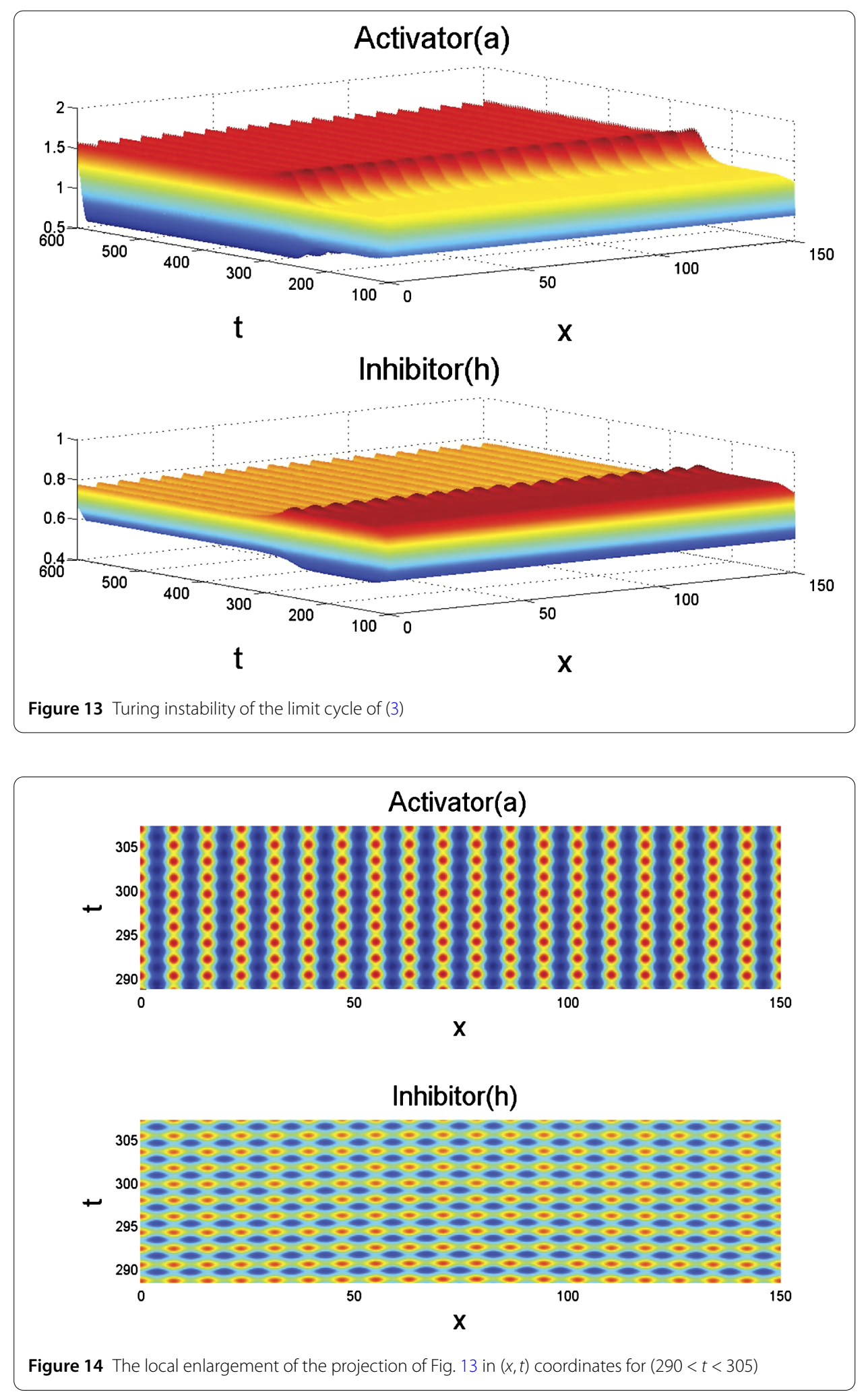

is the region in which the equilibrium may undergo Turing bifurcation. The regions below the line $c=c_{h}$ are also divided into two parts. In the orange region, no Turing patterns will occur. It is the region where the equilibrium is unstable and bifurcates a stable peri- 


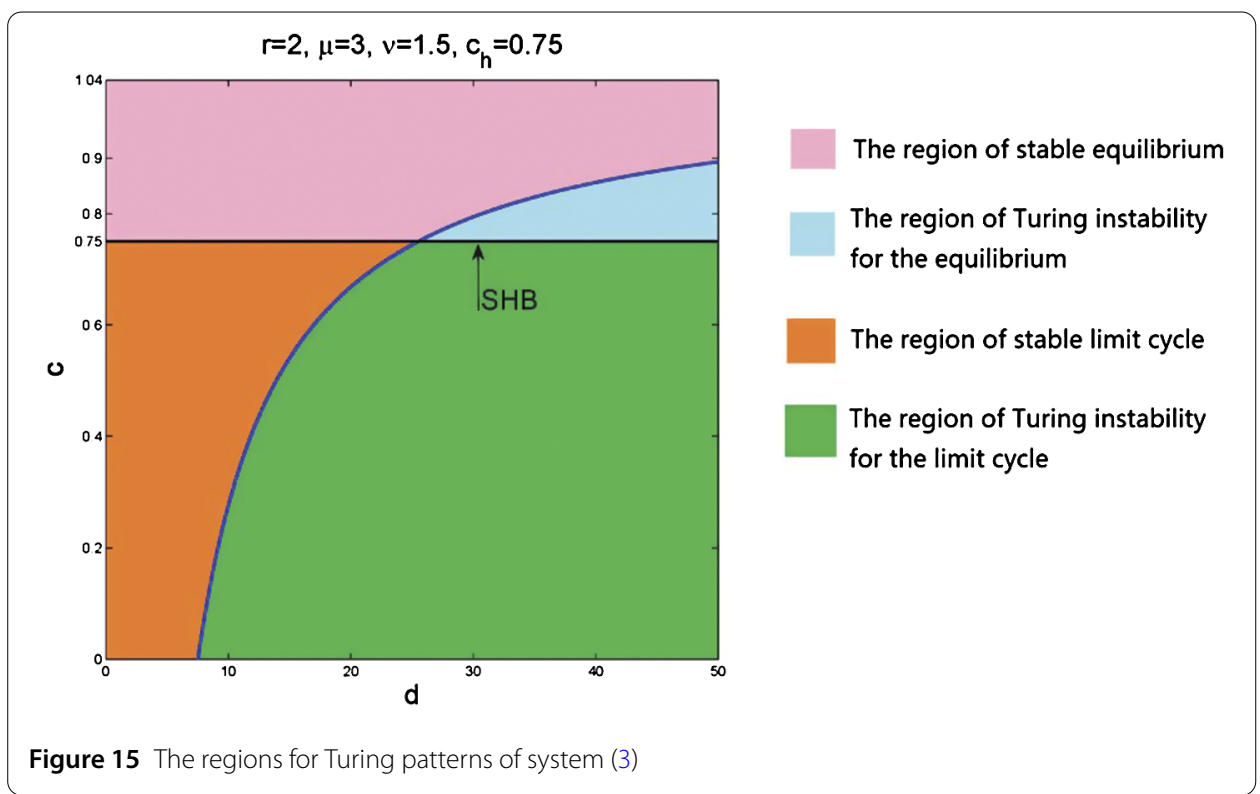

odic solution through Hopf bifurcation. In the green one, the equilibrium is also unstable, whereas the limit cycle experiences a Turing bifurcation and becomes unstable. Turing patterns for the limit cycle may occur in this region.

\section{Acknowledgements}

The authors are grateful to the anonymous reviewers for the helpful suggestions and comments.

\section{Funding}

This work was supported by the National Natural Science Foundation of China (Grant Nos. 10971009, 10771196, 11771033), the National Scholarship Fund (Grant Nos. 201303070222, 201706020203, 201706020094), and the Fundamental Research Funds for the Central Universities.

\section{Competing interests}

The authors declare that they have no competing interests.

\section{Authors' contributions}

All authors contributed equally to writing this paper. All authors read and approved the final manuscript.

\section{Author details}

${ }^{1}$ LMIB \& School of Mathematics and System Science, Beihang University, Beijing, P.R. China. ${ }^{2}$ Department of Mathematics and Statistics, University of North Carolina Wilmington, Wilmington, USA.

\section{Publisher's Note}

Springer Nature remains neutral with regard to jurisdictional claims in published maps and institutional affiliations.

Received: 20 February 2018 Accepted: 4 July 2018 Published online: 13 July 2018

\section{References}

1. Turing, A.M.: The chemical basis of morphogenesis. Philos. Trans. R. Soc. Lond. B 237(641), 37-72 (1952)

2. Murray, J.D.: Mathematical Biology II: Spatial Models and Biomedical Applications. Springer, New York (2001)

3. Gierer, A., Meinhardt, H.: A theory of biological pattern formation. Kybernetik 12(1), 30-39 (1972)

4. Murray, J.D.: Mathematical Biology I: An Introduction. Springer, Berlin (2002)

5. Edelstein, K.L.: Mathematical Models in Biology. SIAM, Berlin (1988)

6. Ruan, S.: Diffusion-driven instability in the Gierer-Meinhardt model of morphogenesis. Nat. Resour. Model. 11(2), $131-141$ (1998)

7. Liu, J., Yi, F., Wei, J: Multiple bifurcation analysis and spatiotemporal patterns in a 1-D Gierer-Meinhardt model of morphogenesis. Int. J. Bifurc. Chaos 20(4), 1007-1025 (2010)

8. Chen, S., Shi, J., Wei, J.: Bifurcation analysis of the Gierer-Meinhardt system with a saturation in the activator production. Appl. Anal. 93(6), 1115-1134 (2014)

9. Li, Y., Wang, J., Hou, X.: Stripe and spot patterns for the Gierer-Meinhardt model with saturated activator production. J. Math. Anal. Appl. 449(2), 1863-1879 (2017) 
10. Maginu, K.: Stability of spatially homogeneous periodic solutions of reaction-diffusion equations. J. Differ. Equ. 31(1), 130-138 (1979)

11. Maginu, K.: Stability of periodic travelling wave solutions with large spatial periods in reaction-diffusion systems. J. Differ. Equ. 39(1), 73-99 (1981)

12. Chen, S., Shi, J.: Global attractivity of equilibrium in Gierer-Meinhardt system with activator production saturation and gene expression time delays. Nonlinear Anal., Real World Appl. 14(4), 1871-1886 (2013)

13. Wang, J., Hou, X., Jing, Z.: Stripe and spot patterns in a Gierer-Meinhardt activator-inhibitor model with different sources. Int. J. Bifurc. Chaos 25(08), 15501081 (2015)

14. Ni, W.: Diffusion, cross-diffusion and their spike-layer steady states. Not. Am. Math. Soc. 45(1), 9-18 (1998)

15. Ward, M.J., Wei, J.: Hopf bifurcation of spike solutions for the shadow Gierer-Meinhardt model. Eur. J. Appl. Math. 14(6), 677-711 (2003)

16. Ward, M.J., Wei, J.: Hopf bifurcations and oscillatory instabilities of spike solutions for the one-dimensional Gierer-Meinhardt model. J. Nonlinear Sci. 13(2), 209-264 (2003)

17. Veerman, F., Doelman, A.: Pulses in a Gierer-Meinhardt equation with a slow nonlinearity. SIAM J. Appl. Dyn. Syst. $12(1), 28-60(2013)$

18. Li, Y., Wang, J., Hou, X.: Stripe and Spot Patterns for General Gierer-Meinhardt Model with Common Sources. Int. J. Bifurc. Chaos 27(02), 1750018 (2017)

19. Takagi, I.: Stability of bifurcating solutions of the Gierer-Meinhardt system. Tohoku Math. J. 31(2), 221-246 (1979)

20. Takagi, I.: Point-condensation for a reaction-diffusion system. J. Differ. Equ. 61(2), 208-249 (1986)

21. Ni, W., Suzuki, K., Takagi, I.: The dynamics of a kinetic activator-inhibitor system. J. Differ. Equ. 229(2), 426-465 (2006)

22. Li, X., Jiang, W., Shi, J.: Hopf bifurcation and Turing instability in the reaction-diffusion Holling-Tanner predator-prey model. IMA J. Appl. Math. 78(2), 287-306 (2013)

23. Yi, F., Wei, J., Shi, J.: Diffusion-driven instability and bifurcation in the Lengyel-Epstein system. Nonlinear Anal., Real World Appl. 9(3), 1038-1051 (2008)

24. Yi, F., Wei, J., Shi, J.: Bifurcation and spatiotemporal patterns in a homogeneous diffusive predator-prey system. J. Differ. Equ. 246(5), 1944-1977 (2009)

25. Marsden, J.E., McCracken, M.: The Hopf Bifurcation and Its Applications. Springer, New York (1976)

26. Wiggins, S.: Introduction to Applied Nonlinear Dynamical Systems and Chaos. Springer, New York (2003)

27. Kuznetsov, Y.A.: Elements of Applied Bifurcation Theory. Springer, New York (1998)

28. Henry, D.: Geometric Theory of Semilinear Parabolic Equations. Springer, Berlin (1981)

29. Crandall, M.G., Rabinowitz, P.H.: The Hopf bifurcation theorem in infinite dimensions. Arch. Ration. Mech. Anal. 67(1), 53-72 (1977)

30. Hassard, B.D., Kazarinoff, N.D., Wan, Y.-H.: Theory and Applications of Hopf Bifurcation. Cambridge University Press, New York (1981)

31. Dilao, R., Sainhas, J.: Validation and calibration of models for reaction-diffusion systems. Int. J. Bifurc. Chaos 8(06), 1163-1182 (1998)

\section{Submit your manuscript to a SpringerOpen ${ }^{\circ}$ journal and benefit from:}

- Convenient online submission

- Rigorous peer review

- Open access: articles freely available online

- High visibility within the field

- Retaining the copyright to your article

Submit your next manuscript at $\gg$ springeropen.com 